\section{General principles and overview of vascular contrast-enhanced ultrasonography}

\author{
Vasileios Rafailidis, Dean Y. Huang, Gibran Timothy Yusuf, Paul S. Sidhu \\ Department of Radiology, King's College London, King's College Hospital, London, UK
}

Ultrasonography (US) is the first-line modality for the evaluation of vascular pathology. Although well-established for many diseases, US has inherent limitations that can occasionally hinder an accurate diagnosis. The value of US was improved by the introduction of microbubbles as ultrasonographic contrast agents (UCAs) and the emergence of contrast-enhanced ultrasonography (CEUS), following the introduction of second-generation UCAs and the emergence of modern contrast-specific techniques. CEUS offers valuable information about vascular disease, both on a macrovascular and a microvascular level, with well-established applications for carotid disease, post-interventional follow-up of abdominal aortic aneurysms, and the assessment of portal vein thrombosis. The purpose of this review is to discuss the principles of CEUS and to present an overview of its vascular applications.

Keywords: Ultrasonography; Carotid artery diseases; Aorta; Atherosclerosis; Aneurysm

\section{Introduction}

Ultrasonography (US) is at the forefront of imaging of vascular disease, as it is a ubiquitous, costeffective, well-tolerated and safe modality. An essential feature of US is its multifaceted nature, as it can provide anatomic information with B-mode and physiologic information with color Doppler, power Doppler and pulsed-wave Doppler techniques, making it indispensable for the assessment of vascular pathology. The multi-parametric nature of US was further advanced by the introduction of new technologies including elastography and contrast-enhanced ultrasound (CEUS), which are encompassed by the term multi-parametric ultrasound [1,2]. From the report of intravascular "bubble clouds" after the intra-aortic catheter injection of saline by Gramiak and Shah in 1968, impressive advances have been made in the field of CEUS, both in terms of microbubble structure and contrastspecific US techniques [3].

The added value of CEUS lies in compensating for the inherent limitations of Doppler techniques, including a lower signal-to-noise ratio, lower sensitivity for slow flow (particularly in deeper vessels) and technical artifacts such as Doppler angle dependence, aliasing and overwriting artifacts. As a result, CEUS not only significantly improves blood flow visualization and wall delineation, but also demonstrates microvascularity. Currently, an increase of more than $30 \mathrm{~dB}$ can be achieved in the echogenicity of blood after administration of an ultrasonographic contrast agent (UCA), and even single microbubbles can be visualized in circulation at the capillary level [4,5]. The introduction of second-generation UCAs in 2001

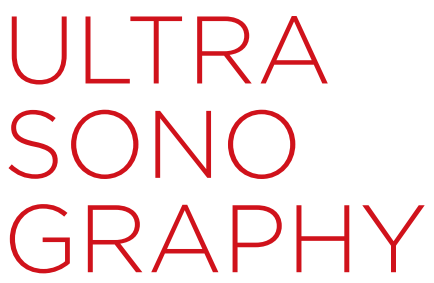

\section{REVIEW ARTICLE}

https://doi.org/10.14366/usg.19022 pISSN: 2288-5919 • elSSN: 2288-5943 Ultrasonography 2020;39:22-42

Received: April 19, 2019

Revised: April 19, 2019

Accepted: June 25, 2019

Correspondence to: Vasileios Rafailidis, MD, MSc, PhD, EDiR, Department of Radiology, King's College London, King's College Hospital, Denmark Hill, London, SE5 9RS, UK

Tel. +44-30-6976161113

E-mail: billraf@hotmail.com

This is an Open Access article distributed under th terms of the Creative Commons Attribution NonCommercial License (http://creativecommons.org/ licenses/by-nc/4.0/) which permits unrestricted noncommercial use, distribution, and reproduction in any medium, provided the original work is properly cited.

Copyright (C) 2020 Korean Society of Ultrasound in Medicine (KSUM)

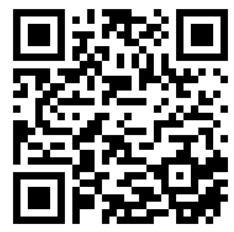

How to cite this article:

Rafailidis V, Huang DY, Yusuf GT, Sidhu PS. General principles and overview of vascular contrast-enhanced ultrasonography. Ultrasonography. 2020 Jan;39(1):22-42. 
and the emergence and widespread availability of low-mechanical index (MI) contrast-specific techniques revolutionized CEUS and led to an expansion of its applications.

CEUS is already a well-established modality for a series of applications in adult patients, with official guidelines and recommendations available $[5,6]$. An explanation and guidance on how to perform CEUS was recently published by the European and World Federations for Ultrasound (European Federation of Societies for Ultrasound in Medicine and Biology [EFSUMB] and World Federation for Ultrasound in Medicine and Biology) [7]. In this paper, we will discuss the basic principles of CEUS and present an overview of its vascular applications.

\section{Microbubbles: Structure and Principles of Interaction with Ultrasound}

UCA microbubbles consist of two parts: (1) an internal gas encapsulated by (2) an outer shell consisting of phospholipids or albumin. The gases contained in first-generation UCAs, such as Levovist (Bayer Schering Pharma, Berlin, Germany) were small molecules that readily traversed the shell of the microbubble and diffused within the blood. Consequently, the duration of enhancement was brief, leading to the use of more inert, hydrophobic and slowly diffusing gases such as sulphur hexafluoride or perfluorobutane in second-generation UCAs, including SonoVue, which is also marketed as Lumason in the United States (Bracco), Definity (Lantheus Medical Imaging), Optison (GE Healthcare) and Sonazoid (GE Healthcare). Each of these agents has different properties, with some being used exclusively for cardiac applications (Optison) and others only for the characterization of focal liver lesions (Sonazoid). SonoVue is the most frequently used UCA for vascular applications, being the only one licensed for use in macrovascular applications including the cerebral arteries, extracranial carotid or peripheral arteries and the portal vein in adult patients, as well as microvascular applications in the liver and breast.

SonoVue consists of sulphur hexafluoride $\left(\mathrm{SF}_{6}\right)$ and a phospholipid monolayer shell, which is amphiphilic, with a hydrophilic outer side and an inner hydrophobic side. With these properties, the shell can successfully contain the gas inside the microbubble. Importantly, this shell is flexible, allowing for changes in the shape and size of the microbubbles, which is a feature useful for generating a reflective signal on US [4]. The microbubbles contained in SonoVue have a mean size of $2.5 \mu \mathrm{m}$ (ranging from 1 to $10 \mu \mathrm{m}$ ). This is a crucial fact for three reasons: (1) this size is large enough to prevent the microbubble from exiting into the extravascular space, (2) it is small enough to permit passage from the lung microcapillaries (with a mean diameter of $7 \mu \mathrm{m}$ ), and (3) it allows for optimal oscillation in a US field. The resonance frequency of a microbubble is associated with its size, ranging from 1 to $10 \mathrm{MHz}$, covering the frequencies used in diagnostic US. The mean diameter of SonoVue $(2.5 \mu \mathrm{m})$ has a resonance frequency of approximately $4 \mathrm{MHz}$, a frequency used for abdominal applications, which explains the excellent signal produced by microbubbles even with a small dose of UCA [4]. UCAs dissolve some minutes after their intravenous administration, with the internal gas being exhaled by the lungs and the phospholipid shell metabolized by the liver. Since the kidneys are not involved in UCA metabolism, these agents are not nephrotoxic, offering a crucial advantage over computed tomography (CT) and magnetic resonance (MR) imaging contrast agents. Moreover, UCAs have no effect on the thyroid, contributing to their safety profile [6]. A property of UCAs that is crucial for vascular CEUS is their strictly intravascular nature, as they never leave the vascular bed (also termed blood-pool agents), in distinction to CT and MR imaging contrast agents, which typically gradually traverse the vascular wall into the extracellular space, reaching an equilibrium concentration between the intravascular and extravascular space. Microbubbles have the property of being strictly intravascular because their diameter is similar to that of red blood cells, making them unable to pass through the vascular endothelium. An exception to this rule is Sonazoid and Levovist, which are phagocytosed by Kupffer cells in the liver, a property which is not useful in vascular imaging $[7,8]$.

The $\mathrm{MI}$ is an important parameter in CEUS, and is defined as the peak negative pressure divided by the square root of the US frequency. In essence, the MI is an indicator of insonation power (amplitude of the US wave pressure) applied to the microbubbles and tissues within the examination field and is typically displayed on the screen during a US examination. Typical MI values for conventional grayscale imaging are 1.6-2.0, and CEUS can be currently performed with much lower MI values [7-9].

Microbubbles tend to respond linearly at very low MI values, meaning that they reflect at the same frequency that is emitted by the transducer (fundamental). When exposed to higher acoustic pressure, but still at a low Ml, the microbubbles oscillate, which is a nonlinear response, and generate harmonic frequencies. At high $\mathrm{MI}$ values, microbubbles oscillate very strongly and are eventually disrupted, emitting strong signals detectable by the transducer. With flexible shells, UCAs can oscillate at MIs well below the breaking point, allowing for optimal visualization $[4,8,9]$. The nonlinear oscillation, in which the microbubbles periodically change in size, expanding during the negative peak of US beam pressure wave and contracting during the positive peak, can be usefully imaged. The microbubble expansion observed is greater than the contraction, explaining the nonlinear nature of the response. This oscillation results in the generation of frequencies higher than the fundamental 
frequency initially hitting the microbubble, which are termed harmonic frequencies. When SonoVue is exposed to a US beam at 3.5 $\mathrm{MHz}$, the frequencies generated include the sub-harmonic (half the fundamental), the ultra-harmonic (1.5 times the fundamental) and the second harmonic (double the fundamental). Even the destruction of a microbubble exposed to a high $\mathrm{Ml}$ generates detectable highintensity signals, which are only very transient and referred to as stimulated acoustic emission. These signals can be accurately visualized with high sensitivity, even with conventional color Doppler US, but this technique cannot be performed continuously due to the destruction of microbubbles [4].

Static tissues exposed to a low-MI US beam generate linear signals. However, the opposite happens when a higher MI is applied, with static tissues responding non-linearly and producing harmonic frequencies, similar to those originating from microbubbles and thus hampering UCA visualization, which is a further reason for using a low Ml for CEUS examinations [8,9].

The safety profile of UCAs is well established, with no evidence of cardiac, hepatic, thyroid or renal toxicity. Severe adverse reactions occur less frequently than with current $\mathrm{CT}$ contrast agents and at roughly the same rate as with MR contrast agents. In a series of 23,188 patients undergoing abdominal CEUS, the rate of lifethreatening allergic reactions was reported to be $0.001 \%$, with no deaths reported and only two patients complaining of serious adverse reactions [10]. Anaphylactoid reactions have been reported to affect $0.014 \%$ of patients undergoing CEUS, a rate lower than that of $\mathrm{CT}(0.035 \%-0.095 \%)$ and comparable to that of MR imaging $(0.001 \%-0.01 \%)[10-14]$. UCAs were equally safe for echocardiographic applications $[15,16]$ and for paediatric applications [17]. Despite the favourable safety profile of UCAs, departments where CEUS examinations are performed should be equipped with appropriate equipment and the personnel should be trained to treat allergic reactions [5-7].

\section{Principles of Contrast-Specific Ultrasonographic Techniques}

Based on the US wave-microbubble interactions, there are two ways of visualizing the UCA signal, which also correspond to the type of UCA. First-generation agents were generally imaged with high-MI techniques (high-MI CEUS with MI values $>0.7$ ), resulting inevitably in intermittent scanning and the subsequent early destruction of microbubbles. These techniques deployed the conventional color Doppler and power Doppler techniques. In simple terms, the UCAs were simply administered in order to increase the signal deriving from blood flow and to improve the signal-to-noise ratio, enhancing Doppler signals [18].
Second-generation agents are more stable, allowing continuous scanning, taking advantage of the emergence of low-MI CEUS techniques and evolving into the standard method for CEUS imaging. Current machines can generate adequate images with an $\mathrm{Ml}$ as low as $\leq 0.1$ (reaching 0.05 ) and maintain microbubble preservation $[4,8]$. The pulse-inversion technique is the latest advance in contrast-specific technologies and currently the most commonly used technique. This technique makes use of the different frequencies generated by microbubbles and static tissue to separate them, thereby enabling the exclusive visualization of microbubbles. The transducer initially emits a sequence of two pulses in rapid succession, identical in frequency and amplitude, but the second pulse is $180^{\circ}$ out of phase compared to the first, representing an inverted copy. Static tissue reflects the same frequencies as those received; thus, the two pulses are cancelled and generate no detectable signal in the transducer. Microbubbles, though, generate harmonic frequencies, which are not opposite and thus add together to produce a strong signal (Fig. 1) [6]. The amplitude modulation technique makes use of a series of pulses with different amplitude, whose reflected signals from microbubbles can be selectively detected and visualized. This technique has better depth penetration than the pulse-inversion technique, although with poorer resolution; therefore, it could be preferred for deep blood vessels $[5,7,19-23]$.

In an attempt to minimize the disruption of microbubbles, a useful strategy is to perform continuous imaging for the first 60 seconds (complete arterial phase and part of venous) and thereafter to scan intermittently with short loops at 30-second intervals to observe the wash-out. This technique is more useful for liver applications, as with vascular applications continuous scanning for 60 seconds

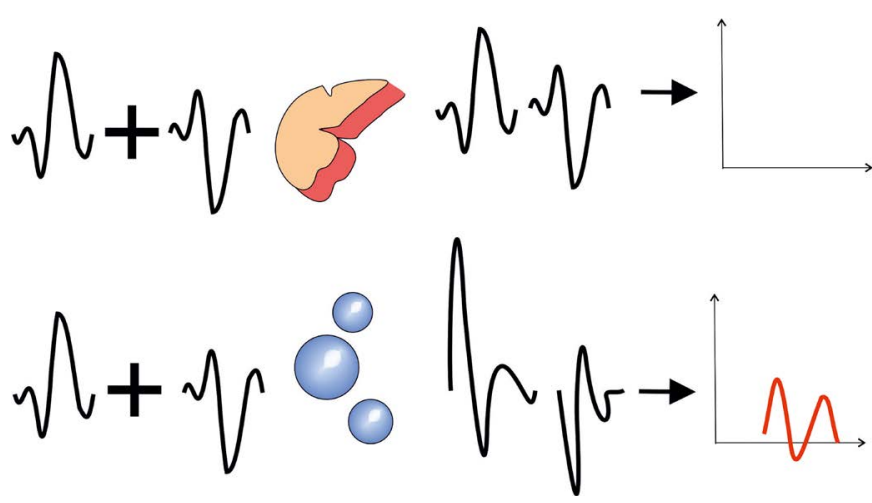

Fig. 1. Schematic diagram showing the pulse-inversion technique. On the top, the static tissue response is illustrated, reflecting the same two inverted pulses that are emitted and thus generating no signal. On the bottom, the microbubbles respond non-linearly, thereby generating harmonic frequencies that, when added, produce a signal detectable by the transducer. 
is usually sufficient to establish the diagnosis and collect relevant clinical information [7].

In low-MI techniques, re-observation of arterial enhancement and the arrival of microbubbles is possible after the destruction of microbubbles with a high-MI pulse, lasting for a couple of seconds. This pulse destroys every microbubble in the plane of insonation and allows the observation of the filling pattern of structures examined for a second or third time. This strategy can be usefully applied in vascular pathology in contexts including (1) re-evaluation of the origin of an endoleak, (2) analysis of enhancement of a plaque, and (3) obtaining evidence of contrast extravasation in vascular injuries.

UCA injection is usually performed with a bolus intravenous injection, followed by $5-10 \mathrm{~mL}$ of normal saline as a flush. The antecubital fossa vein is usually used, although central venous lines and ports may also be used if a peripheral vein cannot be accessed. According to the manufacturer, $2.4 \mathrm{~mL}$ of SonoVue is the recommended dose for vascular applications. Nonetheless, the dose can be adjusted according to the characteristics of the patient and scanning device. For instance, if a high-frequency transducer ( $>10$ $\mathrm{MHz}$ ) is to be used for superficial applications (e.g., scrotal imaging), an increased dose of $4.8 \mathrm{~mL}$ should be considered. In order to avoid microbubble destruction during administration, a 20-gauge cannula should be used and the dose should be administered either directly to the cannula or via the straight line of a 3-way stopcock $[4,7]$. A study found no difference in enhancement when catheters ranging from 18- to 21-gauge were used [24]. A continuous and constant delivery of the UCA (at roughly $1 \mathrm{~mL} / \mathrm{min}$ ) can sometimes be performed in order to lengthen the duration of enhancement and the time of examination; this technique is often used in oncologic applications to assess response to therapy, in myocardial perfusion studies in cardiology and in contrast-enhanced voiding urosonography for the diagnosis of vesicoureteral reflux in children $[4,7]$.

Temporal maximum intensity projection (MIP) is another feature of CEUS, and is useful for demonstrating vascular architecture. In this mode, the US device records bright echoes for a defined time period and accumulates them in order to form detailed images of the macrovasculature and microvasculature. To achieve this, the physician should initially apply a high-Ml pulse in order to disrupt all microbubbles and erase every signal. After this point, every microbubble entering the imaging plane adds its signal to the aggregated image, thereby enabling visualization of the vascular architecture. In essence, the US transducer acts as an "open shutter camera" and produces images similar to those produced with MIP in $\mathrm{CT}$ and MR imaging. Color coding in temporal maps has been recently introduced by some manufacturers to better and more objectively evaluate the arrival of microbubbles using the MIP technique [25].

Useful tips regarding the optimal application of vascular CEUS can be found in Table $1[7,9]$.

\section{Table 1. Tips for performing vascular CEUS}

\begin{tabular}{|c|c|}
\hline & Tip \\
\hline Focus & Place the focus just deep to the target vessel in order to achieve homogeneous energy distribution over the imaging plane. \\
\hline Gain & $\begin{array}{l}\text { The gain should be adjusted at the beginning of the examination so that no UCA signals are lost due to non-detection (too low } \\
\text { gain) or obscuration by noise and erased because of signal saturation (too high gain). The image prior to UCA administration } \\
\text { should be virtually black except for highly echogenic structures like the diaphragm, which are helpful for orientation. }\end{array}$ \\
\hline Dynamic range & $\begin{array}{l}\text { The dynamic range (range of signal intensities displayed) should be wide if fine differences of enhancement are sought (as in } \\
\text { lesion characterization) but can be kept low to make the blood vessels stand out more brightly, with good contrast with the } \\
\text { background. }\end{array}$ \\
\hline Frame rate & $\begin{array}{l}\text { A frame rate of } \geq 10 \mathrm{~Hz} \text { (or frames per second) is useful for assessing wash-in patterns of focal liver lesions or fast-flowing blood } \\
\text { as in some endoleaks, but at the expense of microbubble disruption. }\end{array}$ \\
\hline UCA dose & $\begin{array}{l}\text { Carefully choose the UCA dose, as too high a dose will result in flare (signal saturation) and acoustic shadowing deep to the } \\
\text { microbubbles, whereas too low a dose may cause inadequate enhancement. }\end{array}$ \\
\hline $\mathrm{Ml}$ & $\begin{array}{l}\text { The MI should be properly adjusted. Too high of an MI will cause microbubble disruption, particularly in the near field, whereas } \\
\text { too low of an MI will lead to poor visualization of the far field (e.g., aortic applications). }\end{array}$ \\
\hline Great depth & $\begin{array}{l}\text { CEUS imaging at great depth is always a challenge due to wave attenuation, limiting the depth penetration of low-MI waves. } \\
\text { When possible, a lower insonation frequency can be used (with slightly lower spatial resolution) or different and suitable } \\
\text { imaging windows should be chosen to bring the examined organ closer to the transducer (e.g., the lateral decubitus position } \\
\text { for evaluation of renal vascularity). }\end{array}$ \\
\hline
\end{tabular}

CEUS, contrast-enhanced ultrasonography; UCA, ultrasonographic contrast agent; MI, mechanical index. 


\section{Quantitative CEUS}

CEUS can be subjectively and qualitatively evaluated and interpreted by the physician performing the examination. However, current technology also provides the possibility to quantify the level of enhancement and generate quantitative indexes. Quantitative analysis can be performed either with a bolus injection or with the so called burst-replenishment technique, in which microbubbles are destroyed by a high-Ml pulse after reaching an adequate concentration in the vascular bed and then re-observed during refilling of the field of view. The fact that UCAs remain strictly within the vascular bed means that the calculation of tissue perfusion and microvascularity indices is much more straightforward, compared with agents used for $C T$ and MR imaging that diffuse in the extravascular space $[4,7]$. Quantitative CEUS is performed using time-intensity curve (TIC) analysis, in which the signal intensity of a region of interest is plotted over time. The curve formed represents the pattern of enhancement, increasing with time during the arterial enhancement, reaching a plateau of variable degree and duration and then decreasing during wash-out. Quantitative parameters calculated include peak enhancement, time to peak and area under the curve. An example of how this analysis can help with vascular disease is the quantification of enhancement in carotid plaques in order to quantify intraplaque neovascularization [26] and the TIC analysis of an abdominal aortic aneurysm sac to detect endoleaks after endovascular aneurysm repair $[27,28]$.

\section{CEUS Artifacts}

Many artifacts affect CEUS, some of which also occur in conventional US, whilst others are exclusively encountered in contrast-specific techniques. In the former case, artifacts include increased throughtransmission, mirror artifacts, acoustic shadowing and reverberation (Fig. 2). These artifacts may appear accentuated in CEUS images, since techniques such as spatial compounding and frame averaging are not activated in order to reduce microbubble disruption. In the second category, some artifacts are caused by the inadvertent disruption of microbubbles, such as signal loss due to continuous scanning in one image plane or the near-field signal loss caused by an inappropriately high MI $[9,29]$. In this section, we will focus on CEUS artifacts more closely related to vascular applications of CEUS.

Nonlinear propagation artifacts are a form of pseudoenhancement observed in tissues or the vascular wall situated deep to the vascular lumen. These artifacts are caused by the nonlinear propagation of US waves through a cloud with a high concentration of microbubbles (situated within the vascular lumen) and are visualized as apparent bubble signals in areas with no true enhancement. In vascular applications, these artifacts may lead to a false diagnosis of neovascularization in carotid plaques affecting the distal wall of the carotid. True microbubble signals can be differentiated based on a comparison with the grayscale image, enabling differentiation of artifactual signals, and through observation of enhancement dynamics exhibited by true microbubble movements in all phases. These artifacts can be avoided by avoiding an excessively high dose of UCAs. Another relevant artifact is the visualization on a greyscale image of a hyperechoic structure that is unsuccesfully suppressed and thus appears on a contrast-specific image as bright echoes. This can be misinterpreted as enhancement, although this artifact is easy to identify based on the fact that true microbubbles move, but artifactual echoes are static. In vascular applications, this artifact is frequently encountered due to wall calcifications, which should not be mistaken for plaque ulcerations or neovascularization (Fig. 2) [7,29-31].

In color Doppler US, microbubbles tend to significantly increase the signal intensity of blood vessels and adjacent perfused static tissue. This means that low-intensity signals that were previously filtered now become visible, whilst the intensity of visualized flow signals is markedly enhanced, resulting in excessive color blooming or an overwriting artifact, demonstrating flow signals in areas with no flow (e.g., mural thrombus) and obscuring the surface of plaques and the vascular wall (Fig. 2). Moreover, this artifact may cause the false interpretation of findings as turbulent flow, mimicking stenosis. In the pulsed-wave Doppler technique, early reports showed that peak systolic velocity could be falsely measured (with values of up to $45 \%$ greater than the real values) when the measurement was performed after UCA administration, which was attributed to the increase of signals by the UCA. Nevertheless, this observation was made with older systems, and more recent studies have not been confirmatory $[20,32,33]$. Finally, the pulsedwave Doppler technique may record high-intensity transient signals superimposed on the Doppler waveform, appearing as sharp spikes. These spikes, also audible as crackling sounds, are caused by the disruption of microbubbles by the high-MI pulse used in the Doppler technique (Fig. 2). Based on all these Doppler-related artifacts, it is recommended that Doppler techniques, including color Doppler visualization of blood flow and pulsed-wave Doppler interrogation, should be performed prior to the administration of microbubbles and CEUS should be performed with contrast-specific techniques [20].

\section{Overview of Vascular CEUS}

The role of CEUS in vascular imaging is multifaceted, as the technique is used for the initial diagnostic work-up, guiding of 
interventional treatment and follow-up. Moreover, there are various types of vascular applications, which can be categorized into four groups for educational purposes as follows, described in Table 2 and

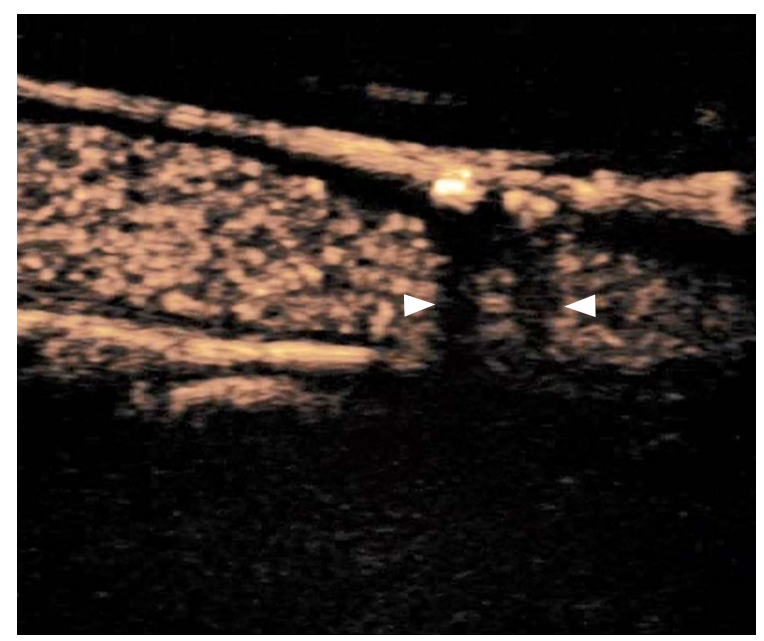

A

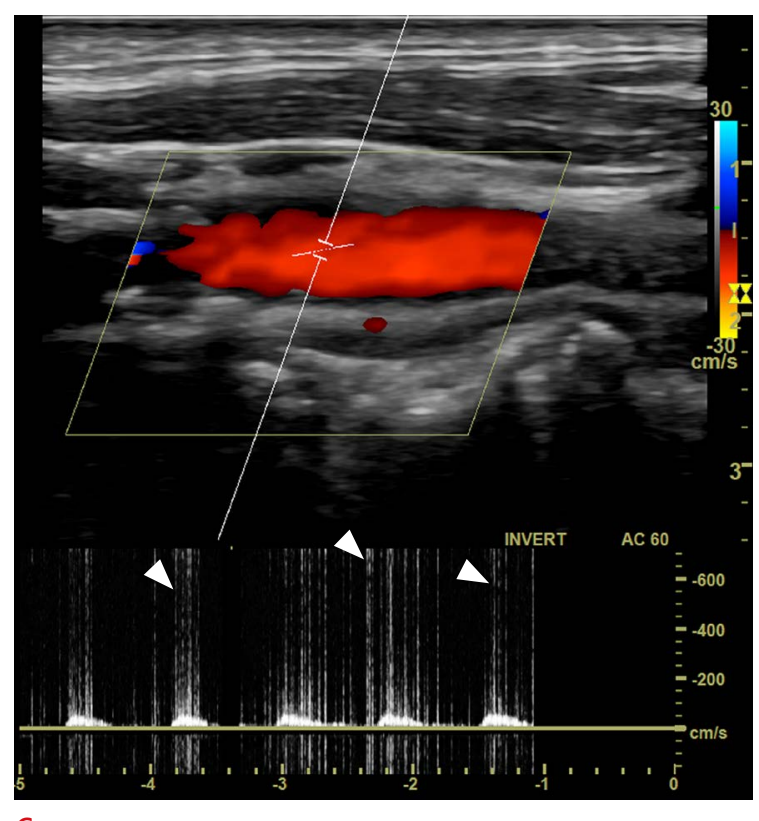

C

Fig. 2. Contrast-enhanced ultrasonography (CEUS) artifacts.

A. Acoustic shadowing (arrowheads) caused by a calcified plaque is shown. B. A color blooming artifact is noted, with color Doppler blood flow signals being enhanced by microbubbles and appearing outside the vascular lumen. C. High-intensity transient signals (arrowheads indicate spikes) are visualized superimposed onto the waveform in the pulsed-wave Doppler technique. D, E. B-mode (D) and CEUS (E) of the same patient show a focal calcification on a plaque (arrows) appearing hyperechoic on B-mode and being projected onto the CEUS image due to unsuccessful suppression. This finding should be carefully recognized to avoid false diagnosis of an ulceration. illustrated in Fig. 3: (1) luminal applications, (2) characterization of intraluminal pathology, (3) characterization of vascular walls, and (4) investigation of potential extravascular leakage. The 2017 version

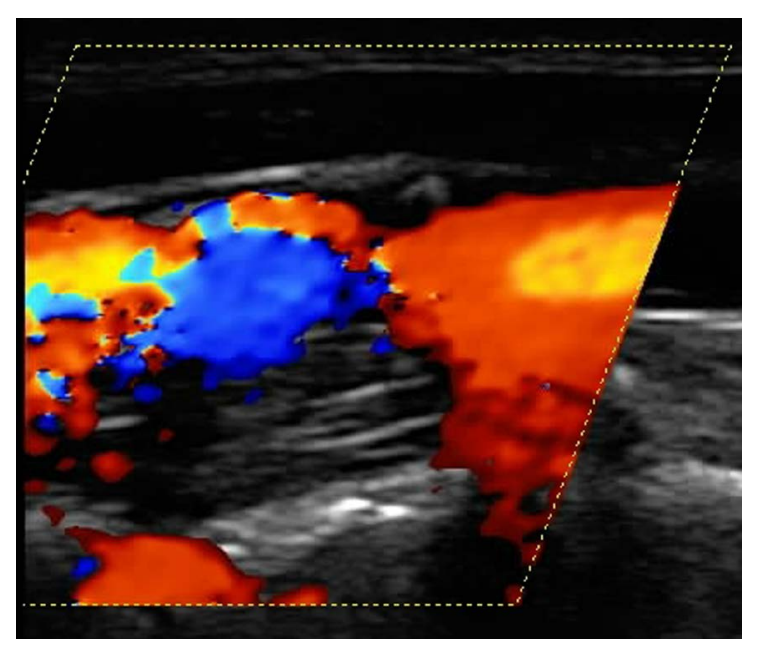

B

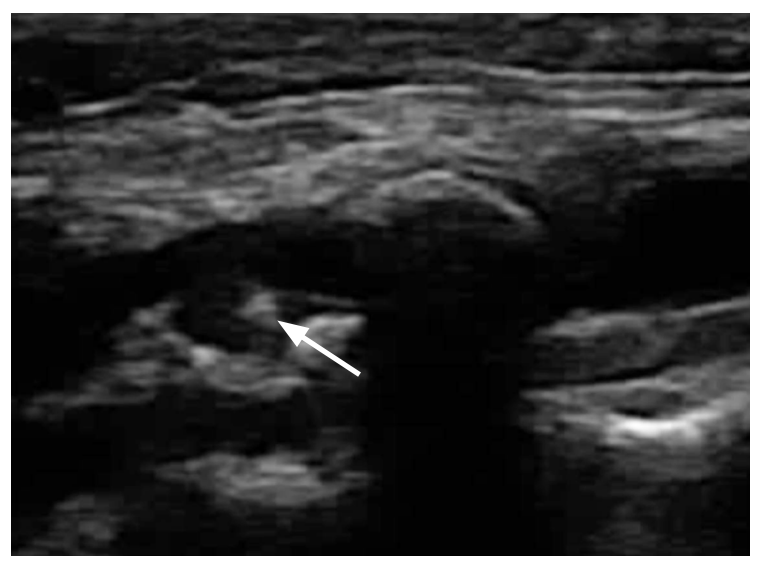

D

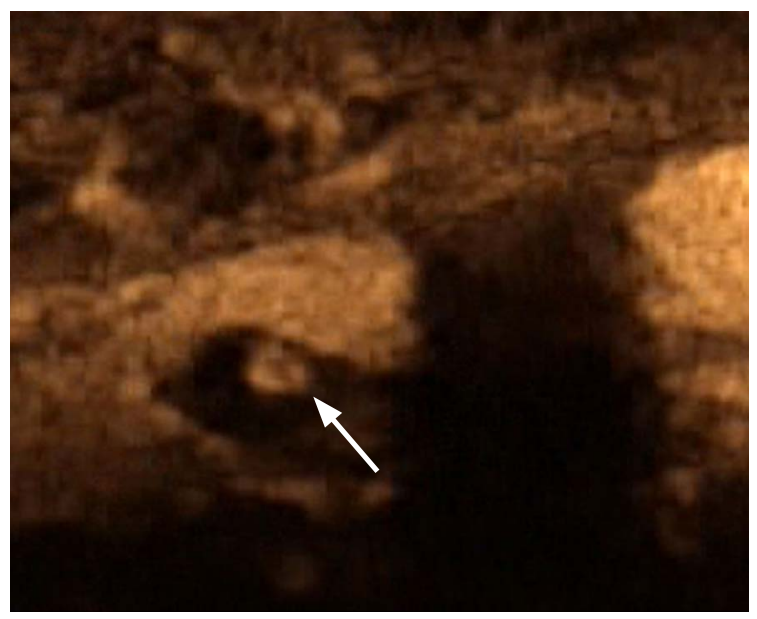

E 
of the EFSUMB guidelines and recommendations on vascular CEUS is presented in Table 3 [6]. Similarly to unenhanced US, CEUS of the carotid and other superficial arteries can be performed with a lineararray transducer of $5-10 \mathrm{MHz}$, whilst the abdominal aorta, portal vein and other abdominal blood vessels can be examined with a convex transducer of $2.5-5 \mathrm{MHz}$ [5].

\section{Carotid Artery}

In carotid artery disease, CEUS has the potential to provide information both on a macrovascular level and a microvascular level. In the first case, CEUS offers improved blood flow visualization and vascular wall delineation, thereby providing accurate grading of
Table 3. The 2017 version of the European Federation for Ultrasound guidelines on the vascular use of CEUS

\begin{tabular}{|c|c|}
\hline Vascular system & Recommendation \\
\hline Carotid arteries & $\begin{array}{l}\text { Differential diagnosis between carotid/vertebral } \\
\text { artery occlusion and severe stenosis } \\
\text { Evaluation of carotid plaque neovascularization, } \\
\text { suggestive of plaque instability }\end{array}$ \\
\hline Abdominal aorta & $\begin{array}{l}\text { Identification of dissection (also in carotid and } \\
\text { vertebral arteries) } \\
\text { Characterization of inflammatory vascular disease } \\
\text { Follow-up of endovascular aortic repair for detection } \\
\text { and classification of endoleaks }\end{array}$ \\
\hline Cerebral vessels & $\begin{array}{l}\text { Improved diagnostic capabilities of contrast- } \\
\text { enhanced transcranial Doppler }\end{array}$ \\
\hline
\end{tabular}

CEUS, contrast-enhanced ultrasonography.

Table 2. Overview of various types of potential vascular CEUS applications grouped into four categories

\begin{tabular}{ll}
\hline Luminal applications & Grading stenosis (e.g., carotid artery) \\
& Occlusion vs. pre-occlusive stenosis \\
& Detection of superficial plaque irregularities and ulceration \\
Characterization of intraluminal pathology & Evaluation of atherosclerotic intraplaque neovascularization \\
& Characterization of portal vein thrombus \\
& Characterization of venous thrombus in peripheral veins \\
Evaluation of the vascular wall & Qualitative and quantitative evaluation of wall vascularity in inflammatory conditions (e.g., aortitis, TIPIC) \\
Investigation of potential extravascular & Endoleak detection after EVAR \\
leakage & Pseudoaneurysm (e.g., peripheral arteries, parenchymal organs) \\
& Extravasation (e.g., aneurysm rupture, vascular injury) \\
\hline
\end{tabular}

CEUS, contrast-enhanced ultrasonography; TIPIC, transient perivascular inflammation of the carotid artery; EVAR, endovascular aortic repair.
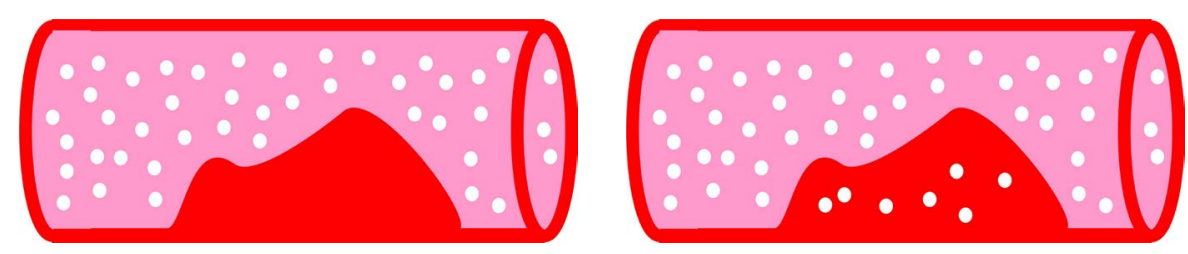

A

B

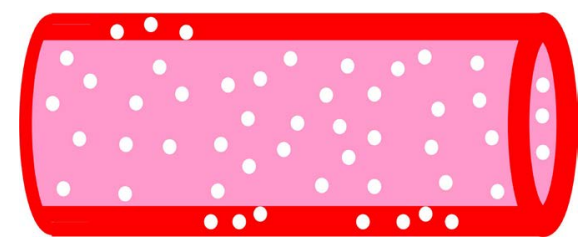

D

$E$

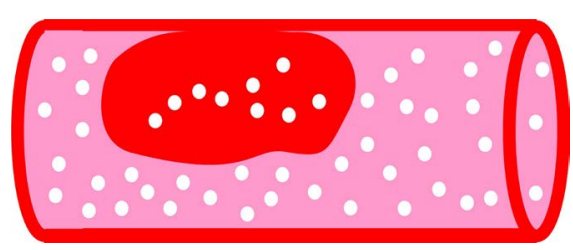

C

Fig. 3. Diagrammatic representation of categories of vascular contrast-enhanced ultrasonography applications.

These applications include (1) luminal applications (A) focusing on improved delineation of the vascular lumen; (2) characterization of intraluminal pathology, such as an atherosclerotic plaque (B) or thrombus (C); (3) characterization of the vascular wall by studying enhancement (D); and (4) investigation of potential extravascular leakage ( $E)$, as in the case of a pseudoaneurysm. 
stenosis, with a strong correlation with conventional angiography for diametric stenosis of the internal carotid artery and with MR imaging for area stenosis [34-36]. Furthermore, CEUS is comparable to $C T$ angiography (CTA) and MR angiography for distinguishing occlusion from pre-occlusive stenosis and outperforms conventional Doppler US, as it is more sensitive in detecting a thread-like lumen in pre-occlusive stenosis [37-39].

In terms of plaque delineation, the use of microbubbles improves delineation of the endovascular border, including the ability to distinguish among plaque, thrombus and vascular wall in the pre-, intra-, and post-stenotic part of the vessel [40]. In this respect, UCAs can be used to accurately diagnose superficial plaque ulcerations, which can be detected with improved sensitivity compared to color Doppler US and represent an essential feature of plaque vulnerability (Fig. 4) [41-44]. On a microvascular level, CEUS can accurately evaluate intraplaque neovascularization, both qualitatively and

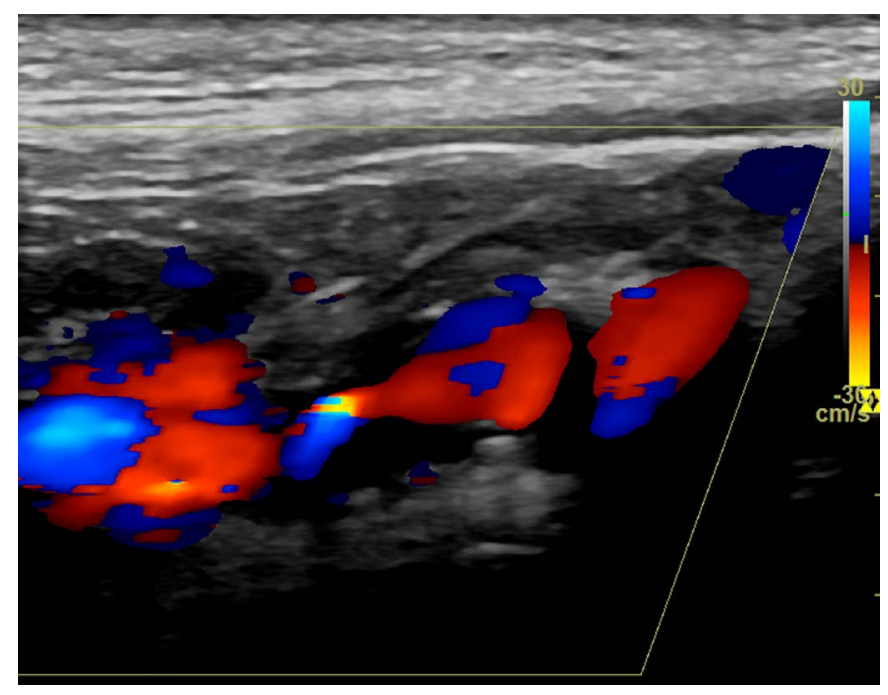

A

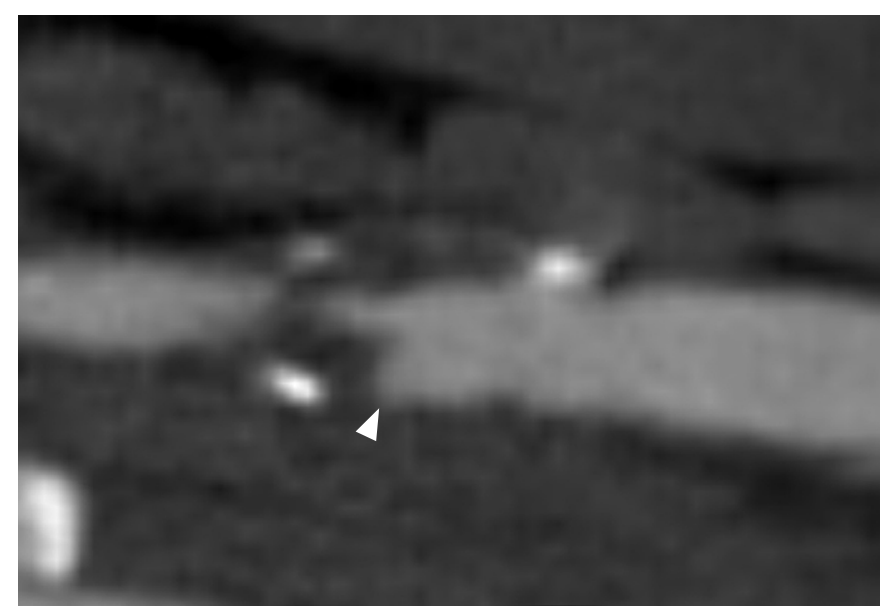

quantitatively using TIC analysis. CEUS findings of neovascularization correlate with histologic findings of neovascularity and inflammation and are predictors of cerebrovascular symptoms, as plaques in symptomatic patients exhibit significantly higher enhancement [41,45-54]. In carotid inflammation including transient perivascular inflammation of the carotid artery (TIPIC) syndrome, CEUS not only better delineated vascular wall irregularities caused by inflammation, but also highlighted wall enhancement consistent with inflammatory vascularization, a marker of disease activity (Fig. 5) $[55,56]$.

Beyond these applications, CEUS is valuable for evaluating restenosis after stenting of the internal carotid artery, improving visualization of the stent lumen [57], and has the potential to identify carotid dissection (Fig. 6) [58]. Although MR imaging is still the reference method, CEUS is able to demonstrate intramural haematoma and, finally, can better delineate fistulae and demonstrate flow within aneurysms or pseudoaneurysms occurring

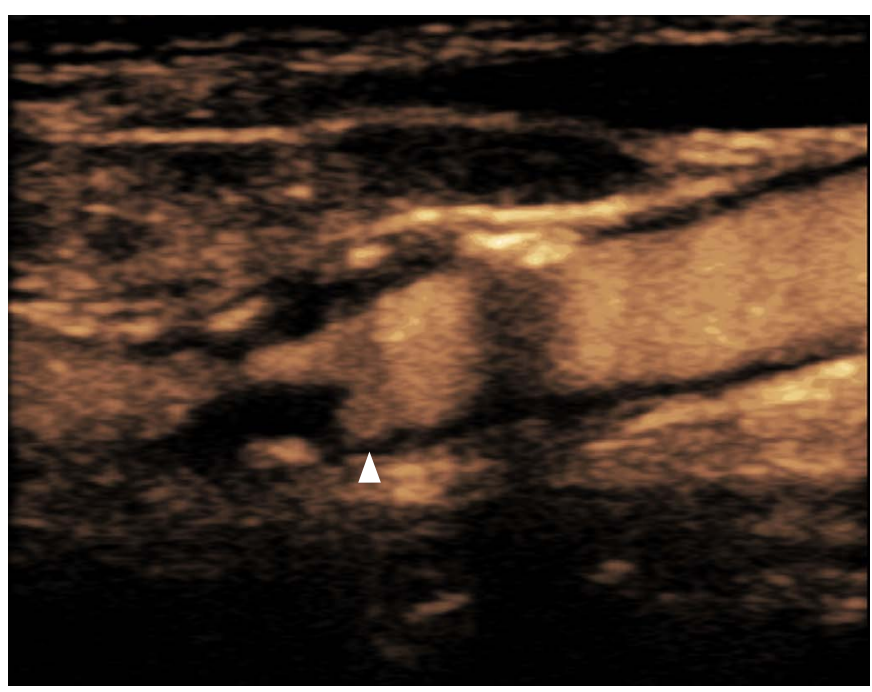

B

Fig. 4. Imaging findings in an ulcerated internal carotid artery plaque.

A. Color Doppler ultrasonography shows a highly stenotic hypoechoic internal carotid artery plaque. The surface of the plaque appears irregular with overwriting artifacts present and color aliasing due to turbulence caused by severe stenosis. B. Contrastenhanced ultrasonography accurately delineates the plaque surface and the vascular lumen, identifying an ulceration (arrowhead) and accurately grading stenosis. C. The ulceration (arrowhead) was also confirmed on computed tomography angiography. 


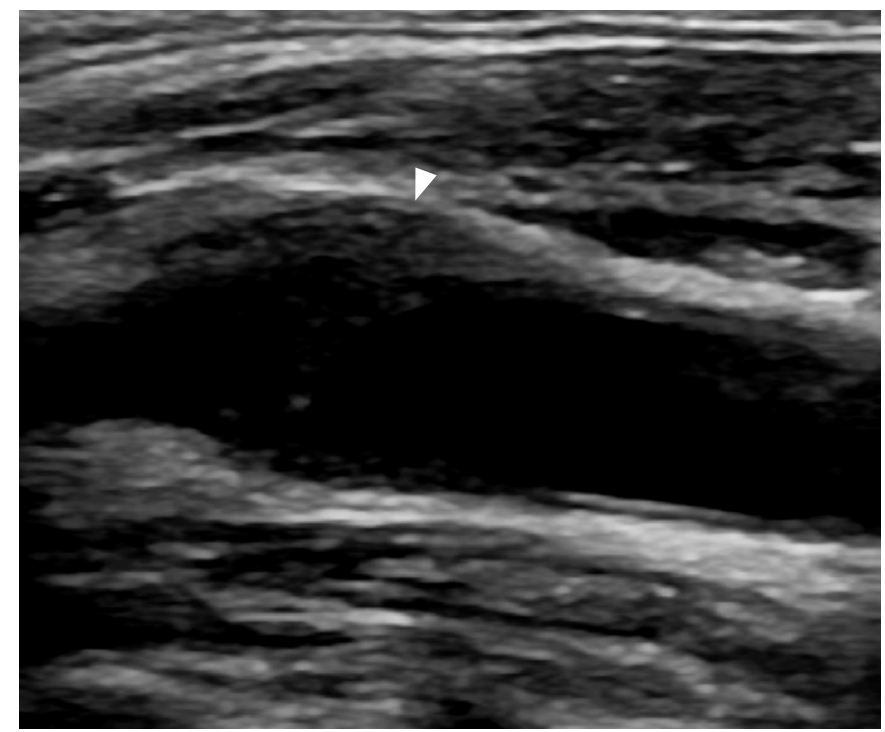

A

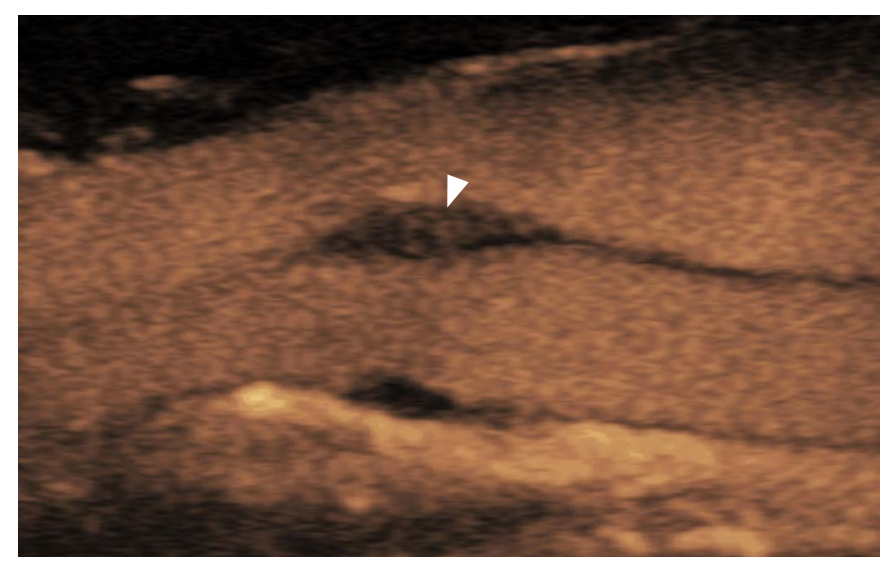

C

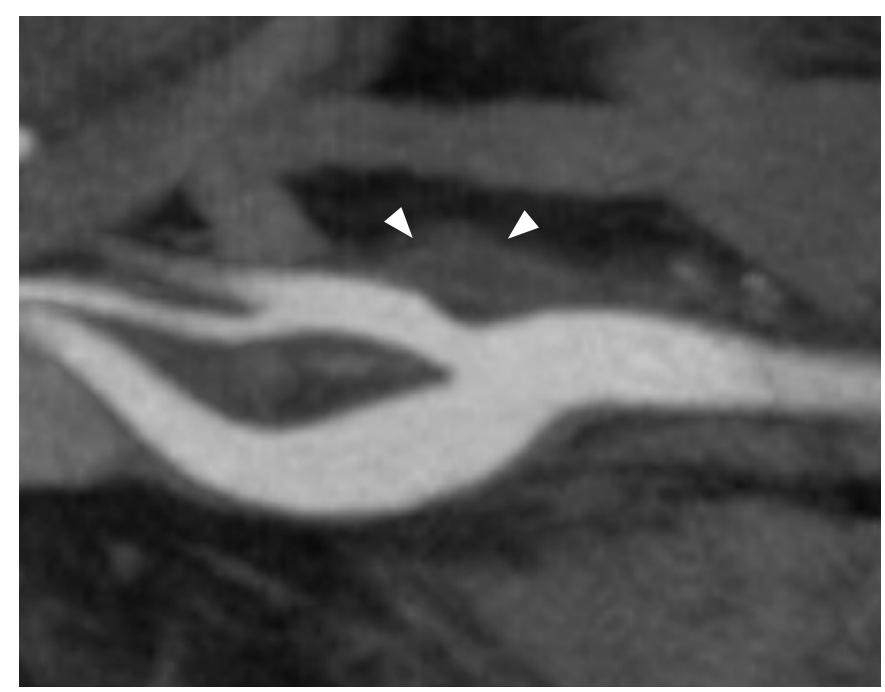

E

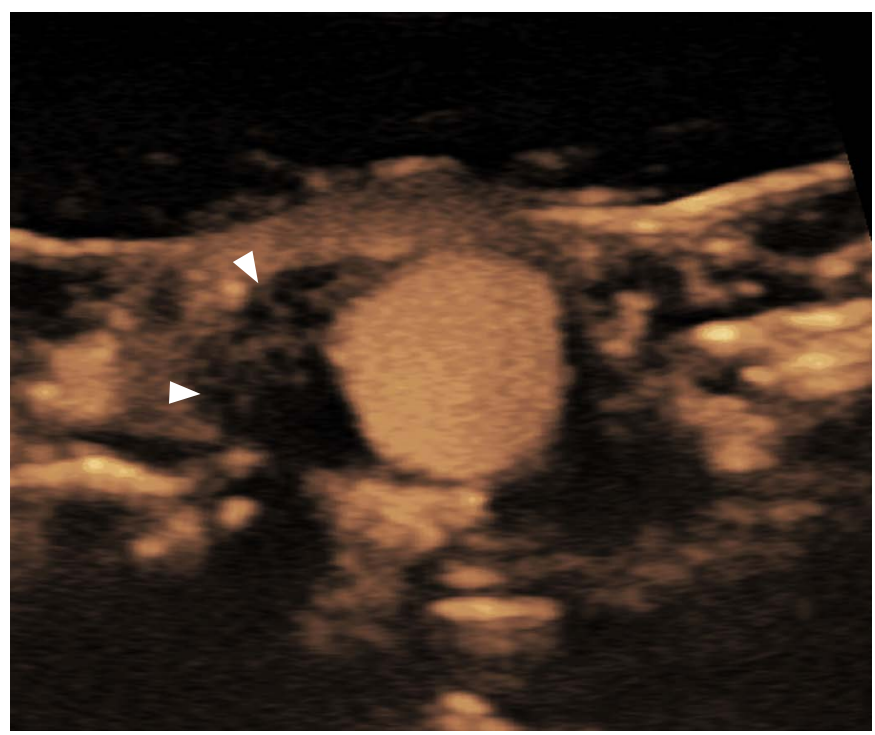

B

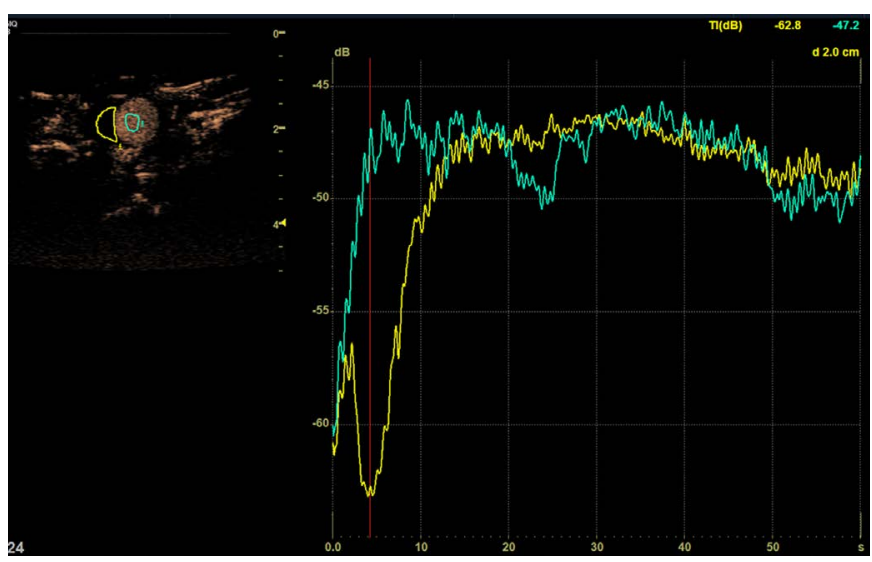

D

Fig. 5. Imaging findings in a 52-year-old man diagnosed with transient perivascular inflammation of the carotid artery syndrome.

A. Longitudinal B-mode image shows hypoechoic mural thickening (arrowhead) eccentrically affecting the origin of the external carotid artery. The lesion was situated exactly at the location where the patient complained of pain. B, C. Transverse (B) and longitudinal (C) temporal maximum intensity projection contrast-enhanced ultrasonography images show intense enhancement of the lesion (arrowheads), a finding in keeping with an inflammatory lesion. D. Time-intensity curve analysis offers the potential to quantitatively analyze the enhancement of the lesion (yellow curve) compared with the lumen for reference (green curve), which is information useful for initial characterization and follow-up after conservative treatment. E. Computed tomography angiography confirmed the findings (arrowheads), although quantitative information about enhancement is not possible with the level of accuracy and duration provided by CEUS. 
as a post-interventional complication $[40,59]$.

\section{Aorta}

CEUS can readily evaluate an aortic aneurysm, whilst also detecting aortic aneurysm rupture by demonstrating microbubble extravasation, although CTA remains the standard method for this diagnosis [60]. In the setting of inflammatory abdominal aortic aneurysm, CEUS can depict enhancement of the aneurysm wall, caused by inflammatory vascularity [61]. As a result, CEUS can discriminate between covered rupture of an aneurysm, showing mural thickening with no enhancement, and inflammatory aortic aneurysm, exhibiting marked enhancement of the vascular wall [62]. Similarly to the carotid arteries, dissection of the abdominal aorta can be readily visualized with CEUS, with higher accuracy than color Doppler US, with particularly improved sensitivity for slow flow, which is commonly encountered in false lumens. Microbubbles are also helpful in differentiating a true lumen from a false lumen, since the first enhances earlier than the second. Importantly, CEUS

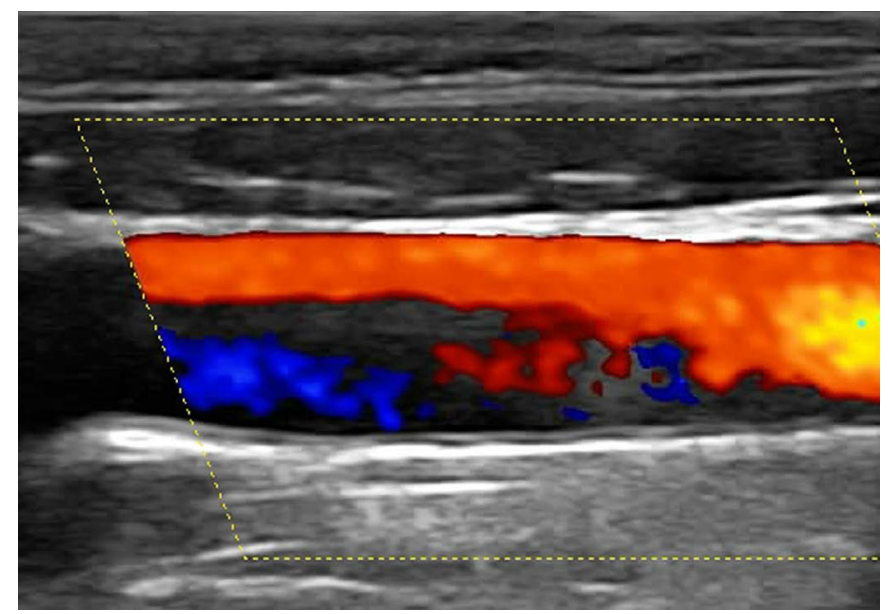

A

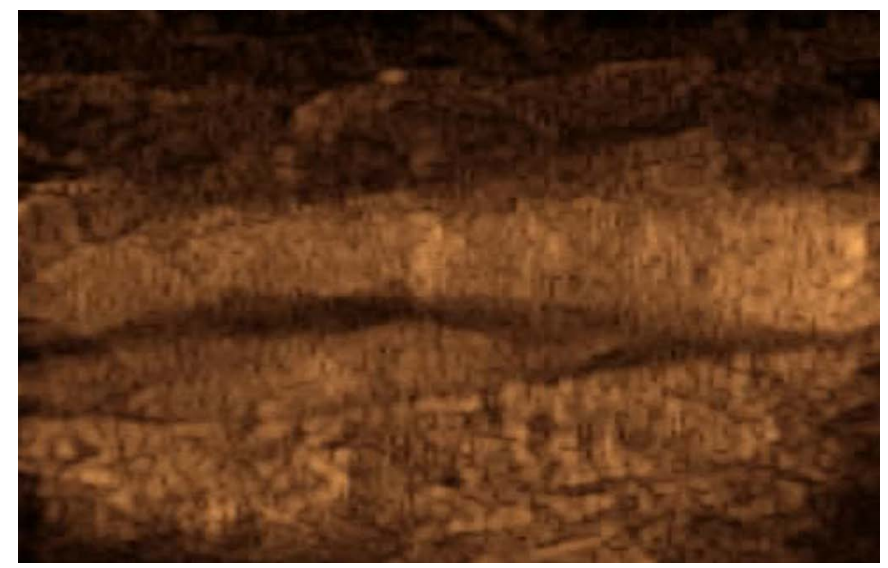

C offers the opportunity to assess tissue perfusion during the same examination, readily demonstrating parenchymal organ ischemia as a complication of dissection [62,63].

After endovascular aortic repair (EVAR), endoleak is a common complication, defined as the presence of blood flow outside the stented lumen, remaining within the borders of the aneurysmal sac and within the thrombotic material filling the latter. Based on the origin of the endoleak, five different types have been documented. A type I endoleak is a high-flow endoleak originating from the proximal (la) or distal (Ib) end of the stent and into the aneurysmal sac. Type II endoleak is the most common, and may be a high- or low-flow leak originating from the inferior mesenteric artery or the lumbar arteries. It is further classified into type lla if a single vessel is involved or type IIb if two vessels are affected. Type III endoleak is rare, being a high-flow leak originating from a defect in the stent graft. Type IV endoleak is associated with porosity of the stent graft material and is observed during stent implantation, requiring no further treatment. Type $V$ endoleak is associated with

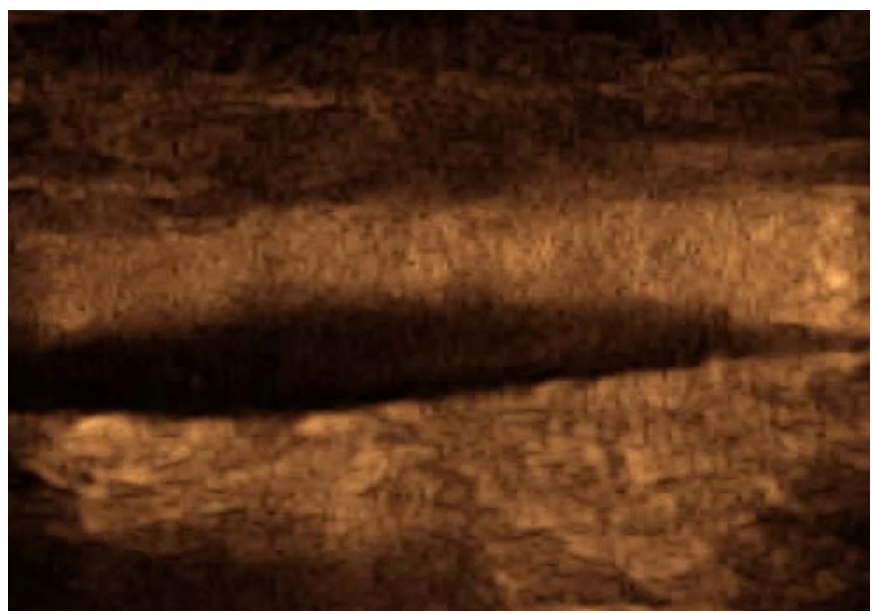

B

Fig. 6. Ultrasonography and contrast-enhanced ultrasonography findings in carotid dissection.

A. Color Doppler image shows narrowing of the common carotid artery lumen by a hypoechoic area possibly exhibiting reversed flow, which was not well visualized tdue to slow flow velocity. B, C. An early contrast-enhanced ultrasonography image (B) and one taken a couple of seconds later (C) show the true lumen enhancing earlier and the false lumen enhancing later. An intimal flap appears anechoic and separates the two lumens. 


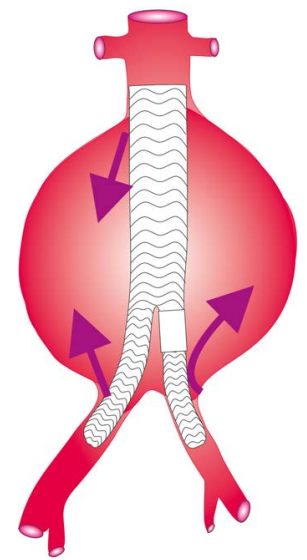

A

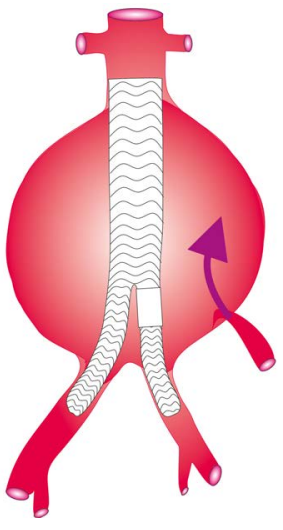

B

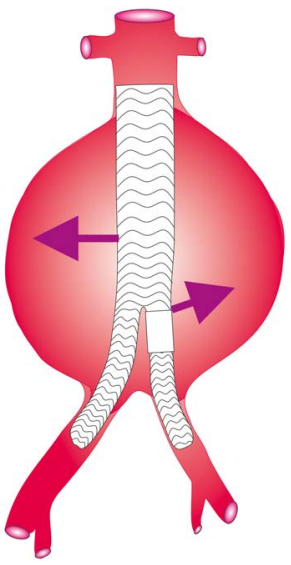

C

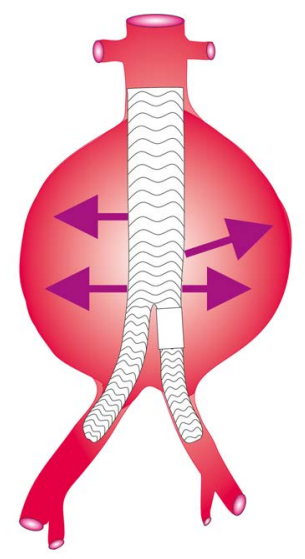

D

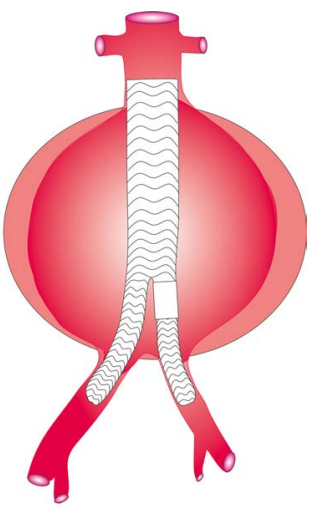

E

Fig. 7. Diagrammatic representation of the five types of endoleaks.

Type I endoleak (A), type II endoleak (B), type III endoleak (C), type IV endoleak (D), and type V endoleak (E) are shown.

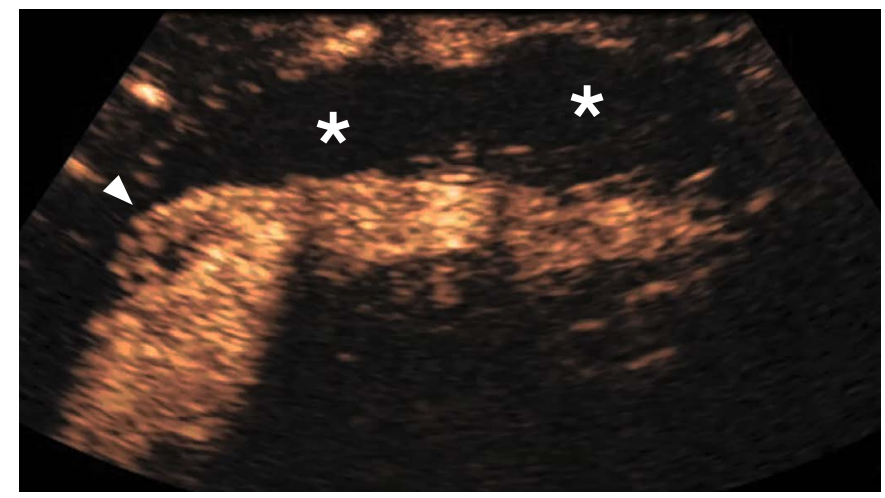

A

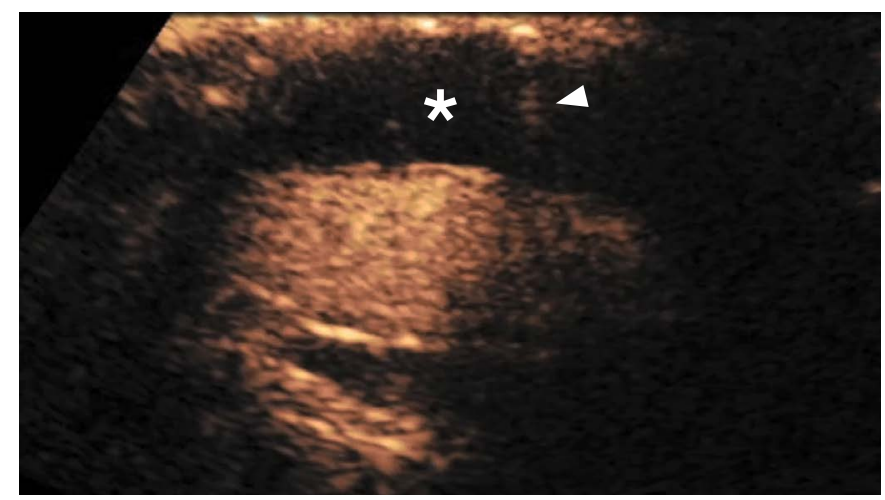

C

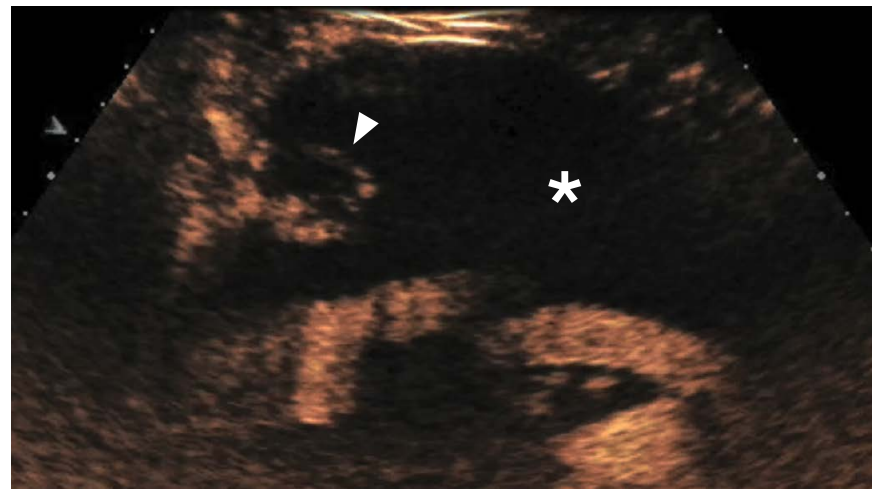

B

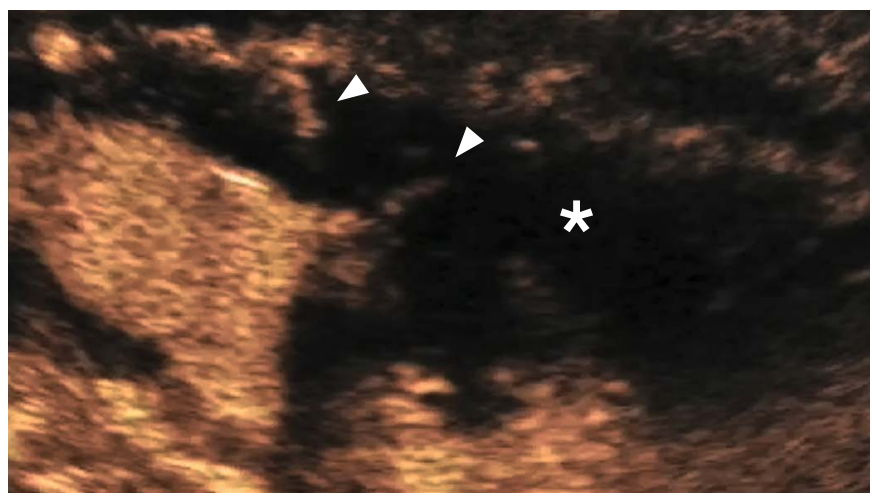

D

Fig. 8. Contrast-enhanced ultrasonography findings of four types of endoleaks.

The asterisks show the occluded aneurysmal sac and the arrowheads the endoleaks. A. A type la endoleak is demonstrated with the endoleak originating from the cephalic end of the stent graft. B. A Type II endoleak is demonstrated with the endoleak originating from a lumbar artery. C. A type III endoleak is demonstrated with the endoleak originating from a defect in the stent graft. D. A type IV endoleak is demonstrated with multiple jets of microbubbles flowing through the fabric of the stent graft. 
an enlargement of the aneurysm sac with no detectable source ("endotension") (Fig. 7) [62].

This is a clinically significant classification given the different treatment required for different types of endoleaks. Type I and III endoleaks require interventional treatment given their higher risk for rupture, whereas type II endoleaks can be followed up with imaging until spontaneous thrombosis. If spontaneous resolution is not achieved, but an enlargement of the aneurysmal sac of more than $1 \mathrm{~cm}$ is documented, an intervention may be required [62]. CTA is the most commonly used modality for detection of endoleaks, although it entails the disadvantage of ionizing radiation and use of a nephrotoxic contrast agent, which is particularly harmful as there is a lifelong need for imaging of these patients. CEUS can diagnose endoleaks with higher accuracy than CTA and can be used to re-categorize previously diagnosed type $\mathrm{V}$ endoleaks (Figs. 8, 9) $[27,64-69]$. Detection of endoleaks with CEUS is typically performed through a qualitative assessment of enhancement, although quantitative analysis with TIC has also been considered, with promising results $[27,28]$. CEUS could be potentially incorporated into imaging algorithms of endoleak detection as a second complementary step to color Doppler US screening. Further imaging with CTA could be safely deferred if no evidence of endoleak is recorded on CEUS, whereas CTA could be performed in cases with abnormal or equivocal findings.

\section{Hepatic Vessels}

The portal vein can be affected by thrombosis, the deposition of thrombotic material in any part of the portal venous system, which may be completely or partially occluded. Portal vein thrombosis is encountered in cirrhotic livers with a prevalence of $0.6 \%-11 \%$ [70]. The thrombus may be bland, usually being silent and having no clinical importance, but it also may be malignant, almost always

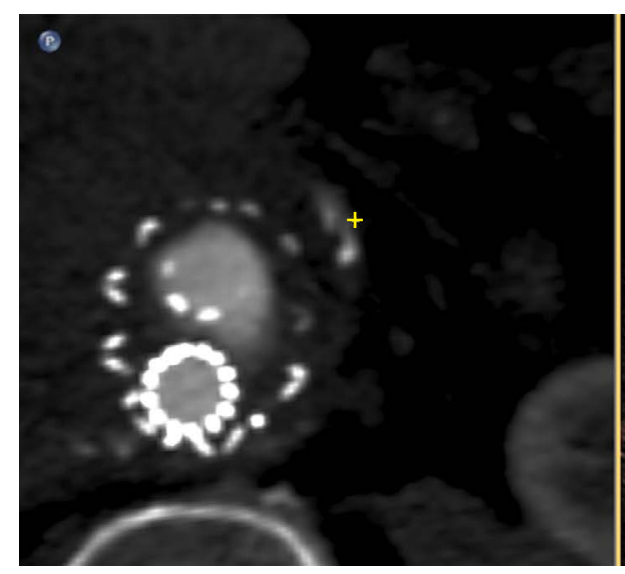

A
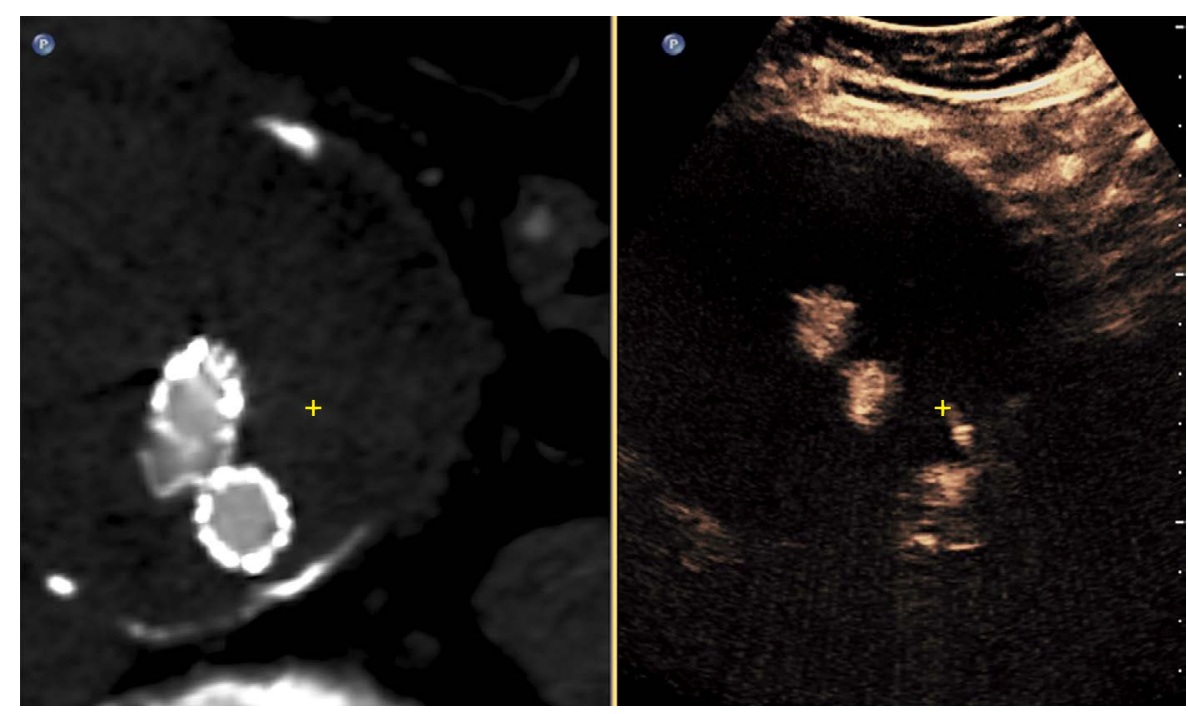

B

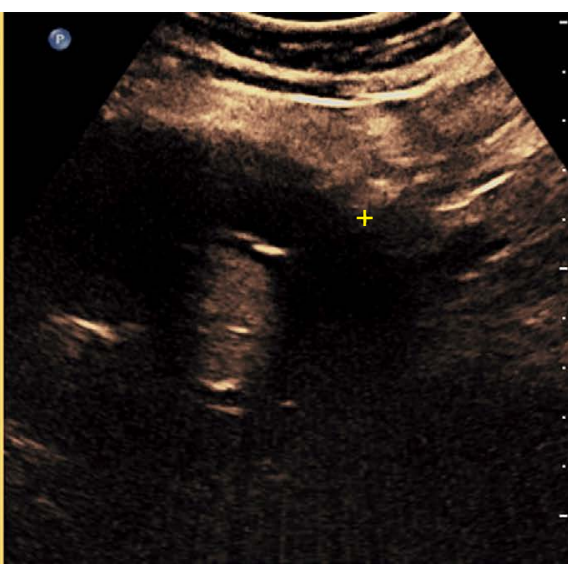

e-ultrasonography.org
Fig. 9. Fusion contrast-enhanced ultrasonography (CEUS)-computed tomography angiography (CTA) technique in a postendovascular aortic repair case of aortic imaging.

A. Registration image shows the same mural calcification in both techniques (yellow cross mark). B. A different fusion image shows a type II endoleak on CEUS (yellow cross mark), possibly originating from a lumbar artery, which was not detected on CTA. 
associated with hepato-cellular carcinoma of the liver. The latter finding carries great clinical significance, with alteration of treatment options and upstaging of the disease. Portal vein thrombus is typically hyperechoic, but may be anechoic, thus making the vessel appear normal on the B-mode technique or hypoechoic. Greyscale imaging of the portal vein should be complemented with the color Doppler technique and pulsed-wave Doppler interrogation. No blood flow signals will be detected in case of complete occlusion by thrombus. The detection of blood flow signals with an arterial waveform on spectral examination inside the thrombus is a highly specific, but moderately sensitive sign of malignancy. Continuity of the thrombus with the hepatic tumour is another feature in keeping with a malignant nature that is evident on greyscale imaging.

CEUS is characterized by improved sensitivity for visualization of thrombi and neovascularity (suggesting malignancy) within the thrombotic material. A bland thrombus appears as a filling defect

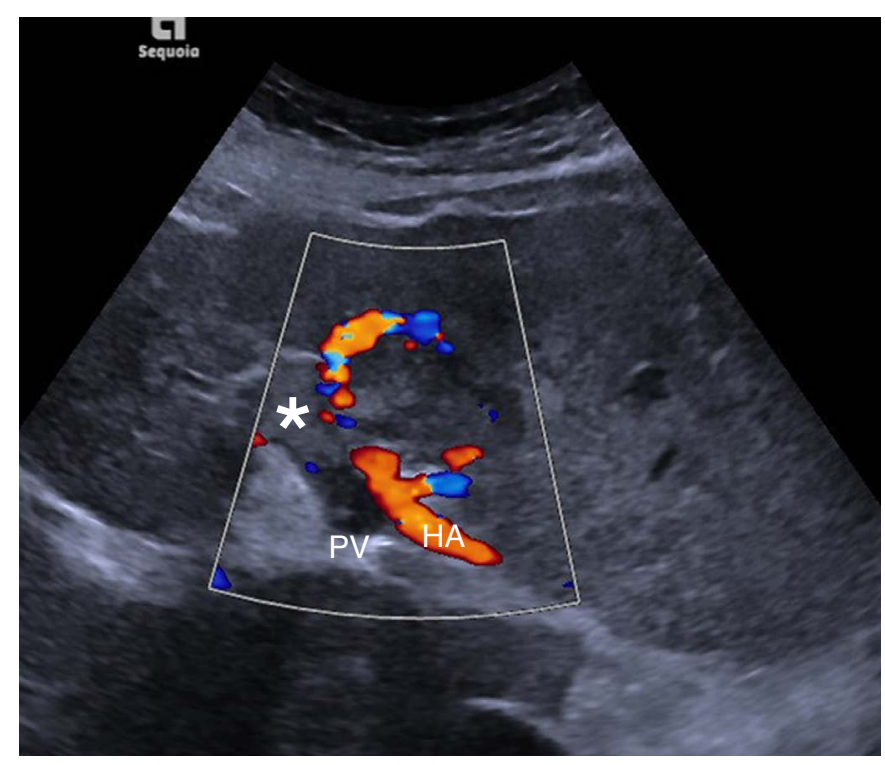

A

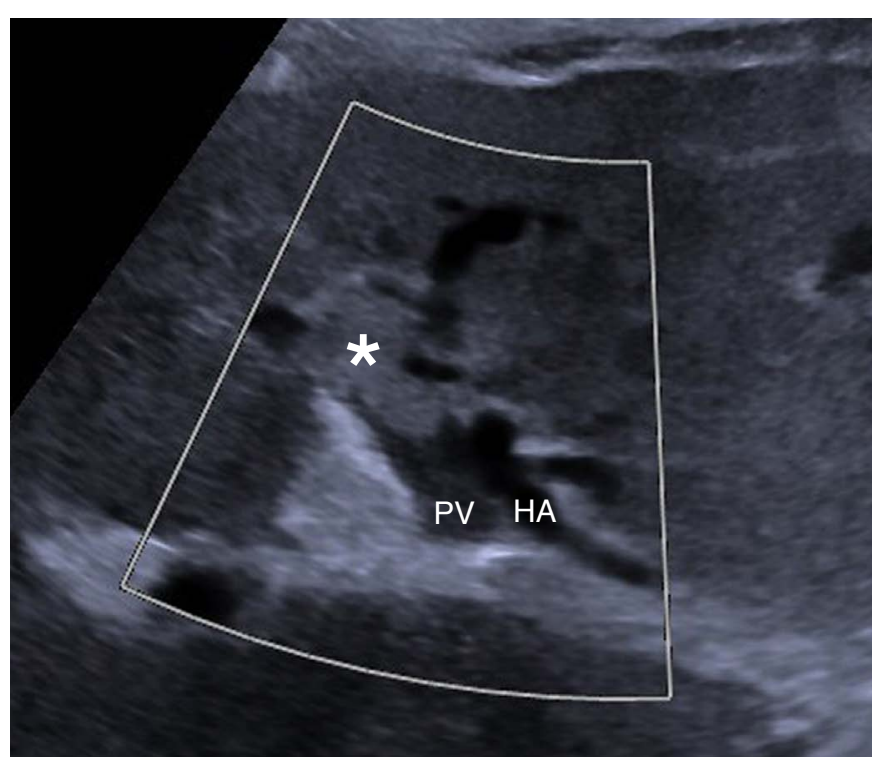

B

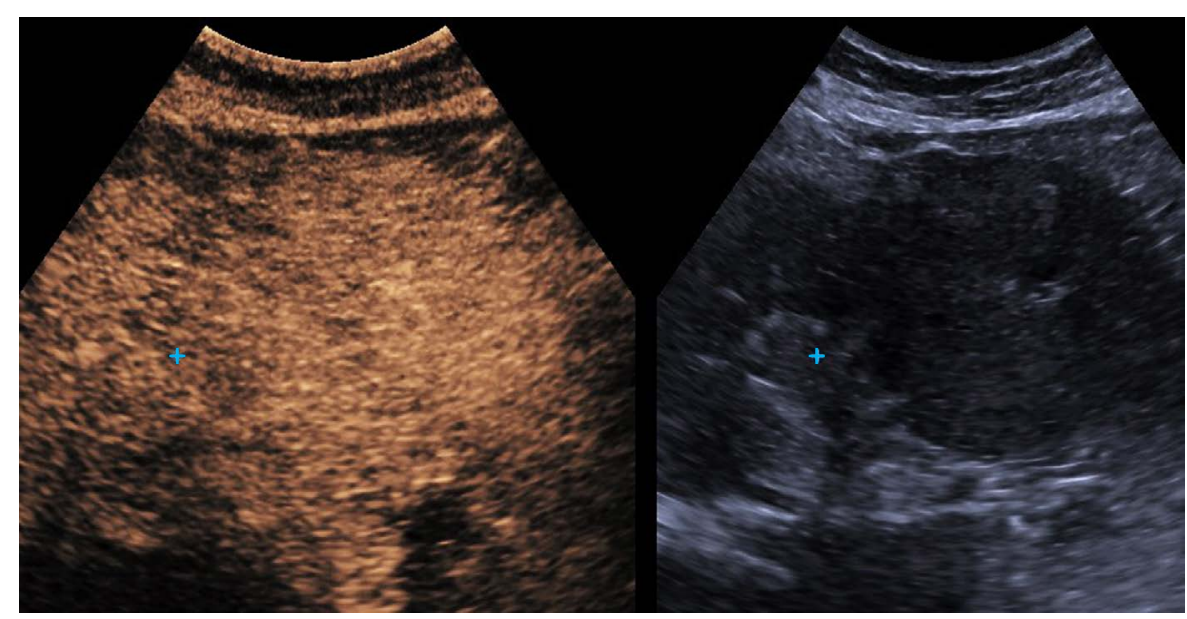

C

Fig. 10. Malignant portal vein thrombosis on conventional ultrasonography and contrast-enhanced ultrasonography (CEUS).

A. Color Doppler ultrasound shows patency of the hepatic artery (HA), but an absence of blood flow signals within the portal vein (PV), part of which appears filled with echogenic material (asterisk) consistent with thrombosis. No definite blood flow signals can be detected within the thrombus, suggesting the diagnosis of bland thrombus. B. A focused Clarify vascular enhancement mode image (Siemens Healthcare) shows a B-mode image of vessels with reduced noise and artifacts and with better definition (asterisk indicates thrombus). C. Portal venousphase CEUS image shows enhancement of the thrombus (blue cross mark) but relative wash-out compared with the hepatic parenchyma, which are findings that establish the diagnosis of malignant thrombosis. 
within the portal vein, avascular in all phases, although most conspicuous in the portal venous phase. A malignant thrombus has the same enhancement pattern as the tumour of origin, including rapid arterial phase hyper-enhancement simultaneously with the hepatic artery and rapid or late and mild portal venous wash-out (Fig. 10).

The thrombus can be targeted for biopsy under US guidance, with an attempt made to take specimens from enhancing regions of the thrombus. CEUS has also been found to be very useful in complementing US-guided biopsy of the thrombus in order to establish the diagnosis of benign or malignant thrombus. The tumour causing the thrombus may not be visible with US, although it can sometimes be detected while scanning the liver in any phase after UCA administration. Washed-out areas detected in the portal venous phase can be observed in the arterial phase (for vascularization) after re-injection to confirm the diagnosis. Compared with $\mathrm{CT}$ and MR imaging, CEUS offers the advantage of continuous and real-time

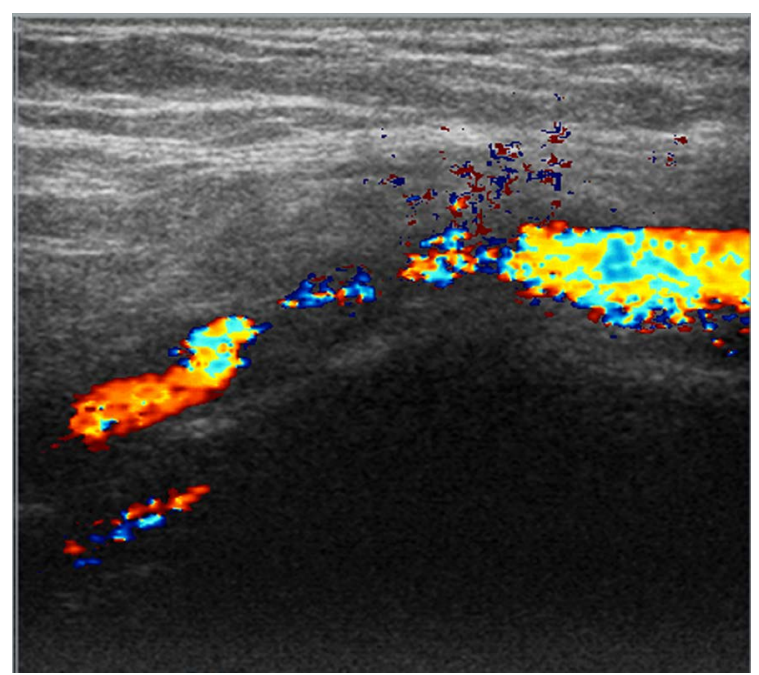

A

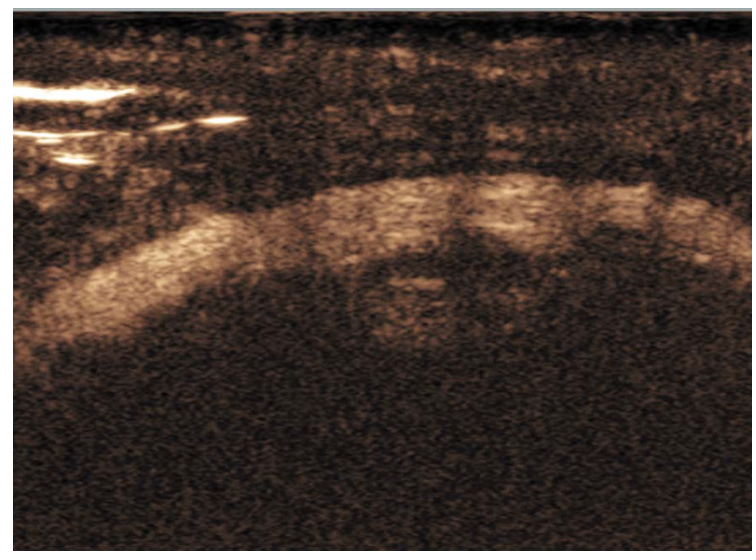

scanning of thrombus vascularity for several minutes, whereas the former techniques typically record a few "snapshots" of thrombus enhancement [70-75].

\section{Peripheral Arteries}

When it comes to peripheral arteries, conventional Doppler US techniques are well-established and CEUS has not demonstrated significant superiority for detecting stenosis, although it can be used to detect complications after interventional procedures, as in cases of femoral artery pseudoaneurysm (Figs. 11, 12). Vascular injury after trauma, along with other causes, may result in the formation of aneurysms or, more commonly, pseudoaneurysms, which potentially require interventional treatment if they become symptomatic and rupture. CEUS not only can detect such a complication, but can also potentially determine the anatomic location of the origin of ongoing haemorrhage (Figs. 13, 14) [76]. In a paediatric population series, pseudoaneurysms were encountered in $17 \%$ of children sustaining

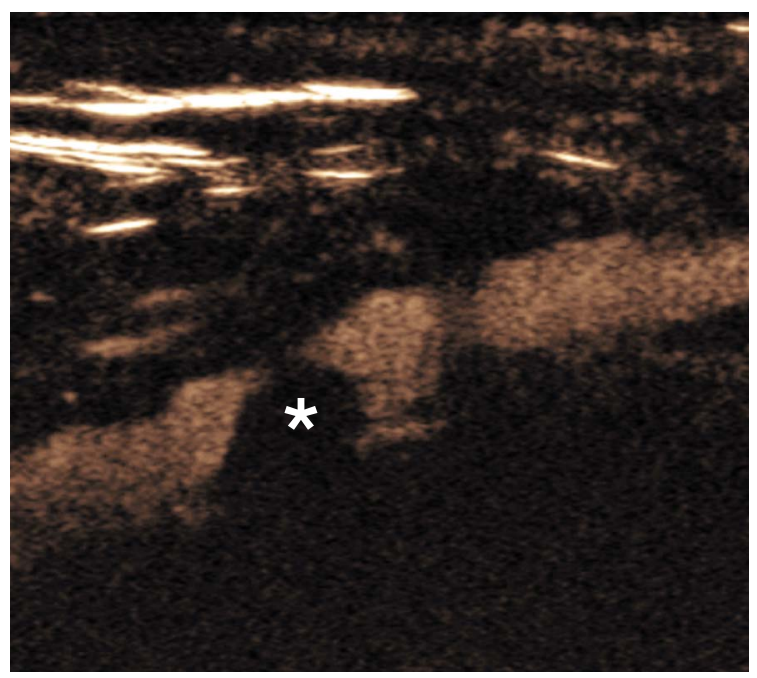

B

Fig. 11. Atherosclerosis of the external iliac artery before and after angioplasty.

A. Color Doppler ultrasonography shows severe stenosis with significant turbulence. Note the presence of aliasing and an overwriting artifact, whilst some parts of the lumen are not filled with Doppler signals due to low sensitivity. B. A contrast-enhanced ultrasonography (CEUS) image accurately delineates the borders of a plaque (asterisk) causing severe stenosis. The lumen is accurately visualized in its pre-, intra- and post-stenotic part. C. A CEUS image following angioplasty confirms the favourable result and the restored patency of the vessel. 
liver or splenic injuries and CEUS was $83 \%$ sensitive and $92 \%$ specific for this diagnosis, offering a valuable alternative to $\mathrm{CT}$ for diagnosis and follow-up of this entity [77].

\section{Conclusion}

CEUS is a well-suited modality for the evaluation of vascular pathology given its favourable inherent characteristics such as the strictly intravascular nature of microbubbles used as UCAs. Physicians performing this technique should be familiar with basic physical principles in order to recognize artifacts, avoid misdiagnoses and correct them by making adjustments to scanning parameters. CEUS has already been studied in many vascular applications such as carotid disease, post-EVAR aortic evaluation and portal vein

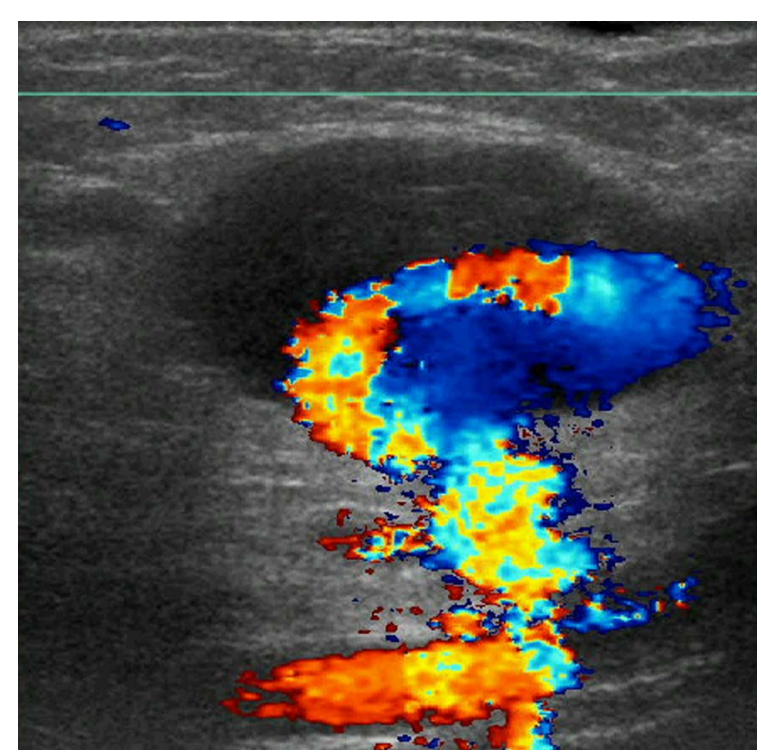

A

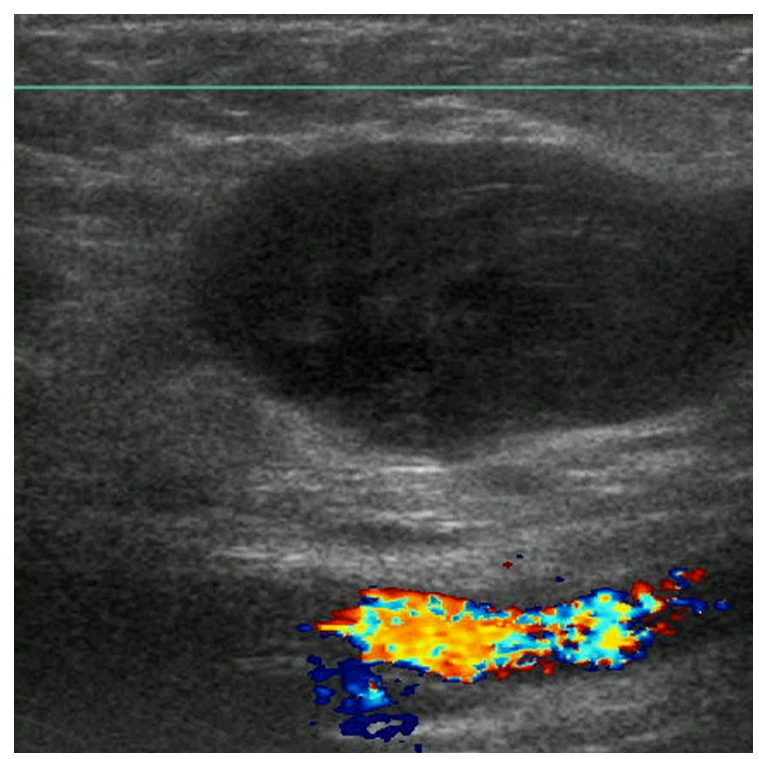

C

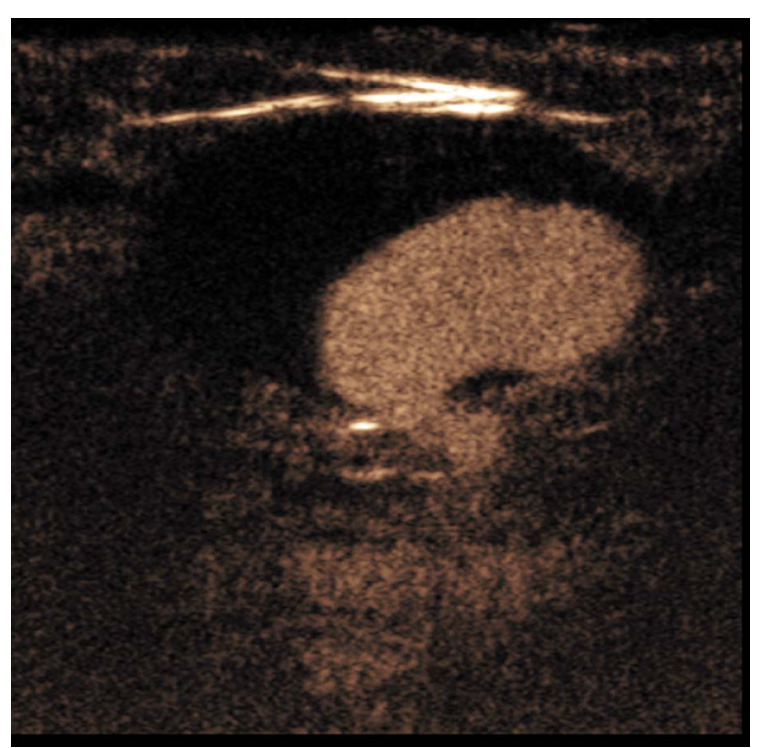

B

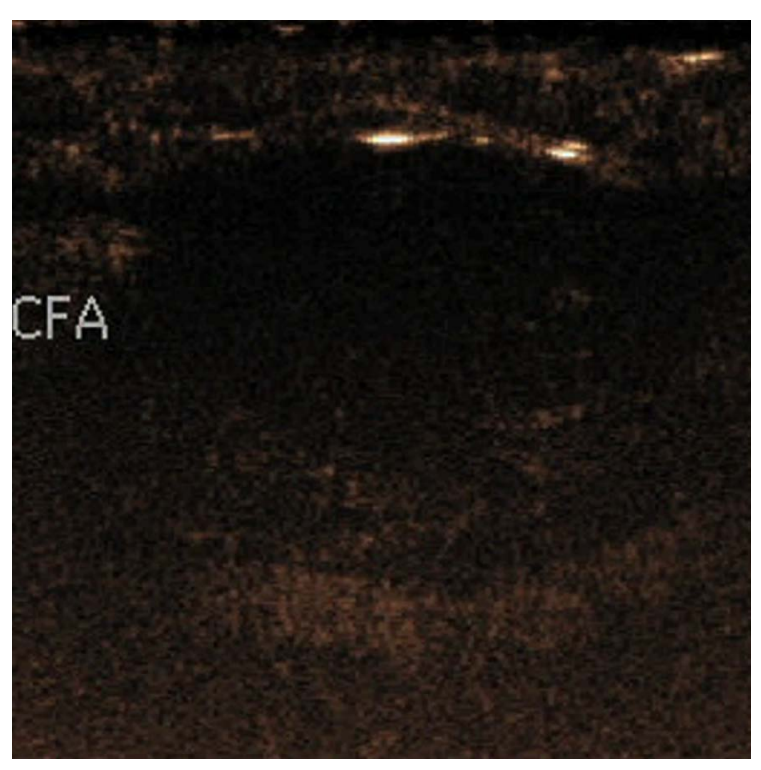

D

Fig. 12. Superficial femoral artery pseudoaneurysm on color Doppler ultrasound and contrast-enhanced ultrasonography (CEUS).

A. Color Doppler ultrasonography (US) shows partial filling of the pseudoaneurysm along with its connection with the vessel of origin. The cavity of the pseudoaneurysm is partially filled with an echogenic thrombus. Color flow reversal is noted, giving rise to a "yin-yang" appearance. Overwriting artifacts obscure the fistulous neck of the pseudoaneurysm. B. A CEUS image shows filling of the patent part of the pseudoaneurysm and accurately delineates its neck. C, D. After thrombin injection, color Doppler US (C) and CEUS (D) show absence of blood flow within the successfully treated pseudoaneurysm. CFA, common femoral artery. 


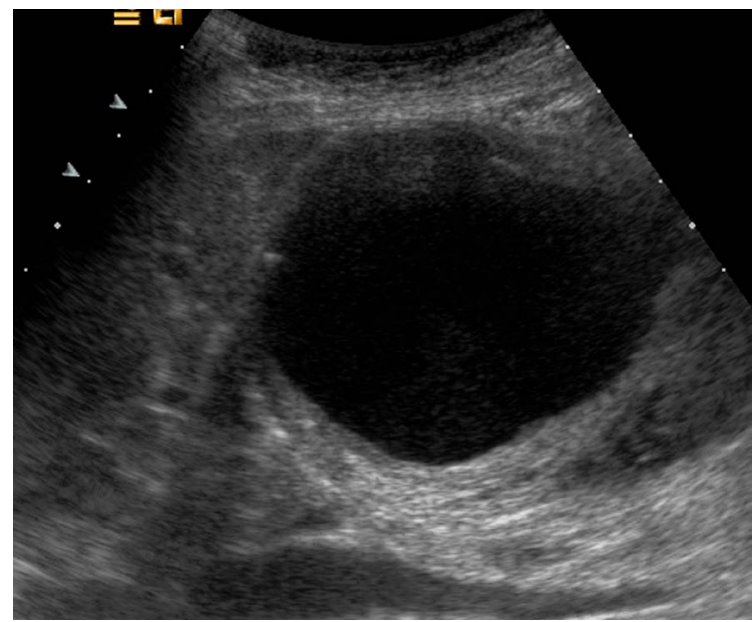

A

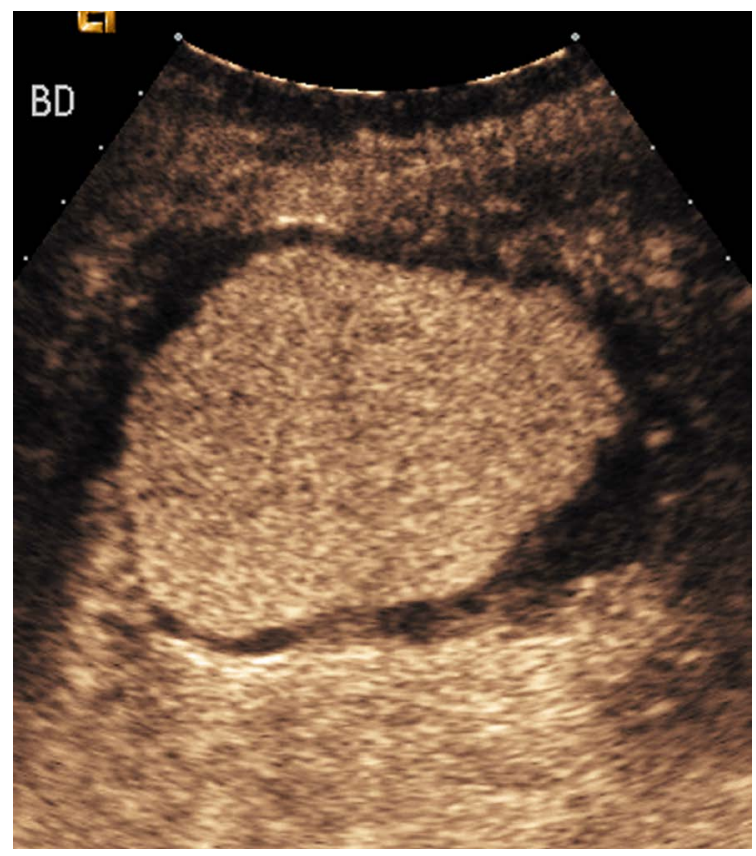

C

Fig. 13. Hepatic artery pseudoaneurysm.

A. A B-mode image shows an enlarged anechoic rounded structure with a thickened wall, situated near the porta hepatis. B. Color Doppler ultrasonography shows the filling of the structure with color Doppler signals, establishing the diagnosis of pseudoaneurysm. Note that parts of the cavity are not filled with signals due to a lack of sensitivity. C. Contrast-enhanced ultrasonography (CEUS) image shows the filling of the pseudoaneurysm with microbubbles except for the limited mural thrombus, which appears anechoic. D. Computed tomography angiography confirms the diagnosis of a hepatic artery pseudoaneurysm, with mural thrombus and calcifications. E. After the placement of a stent (arrowhead), CEUS confirms the exclusion of the aneurysmal sac from circulation by documenting a lack of enhancement.

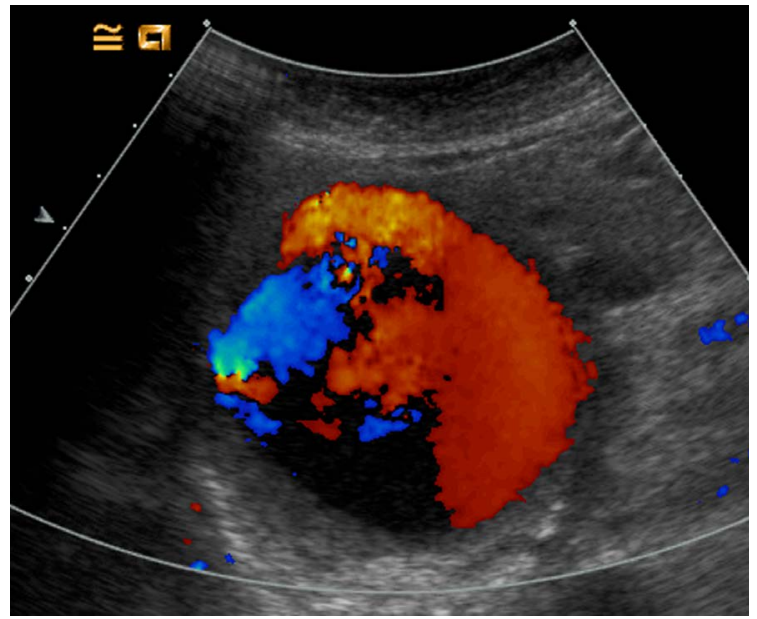

B

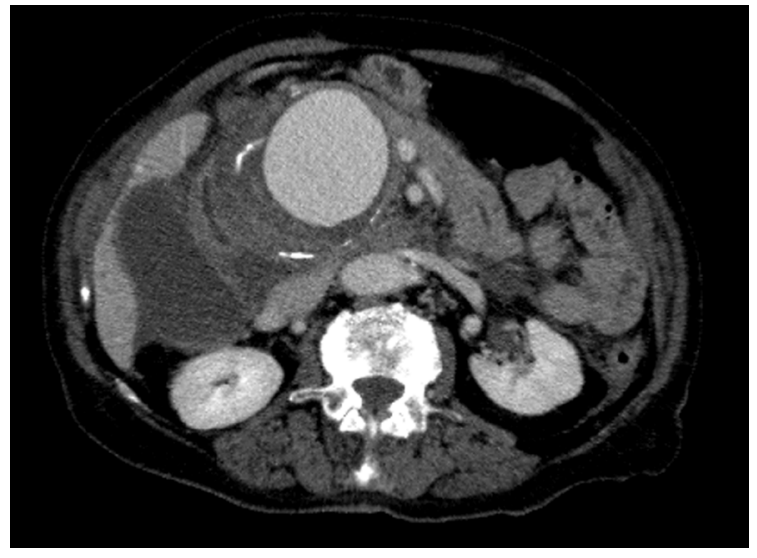

D

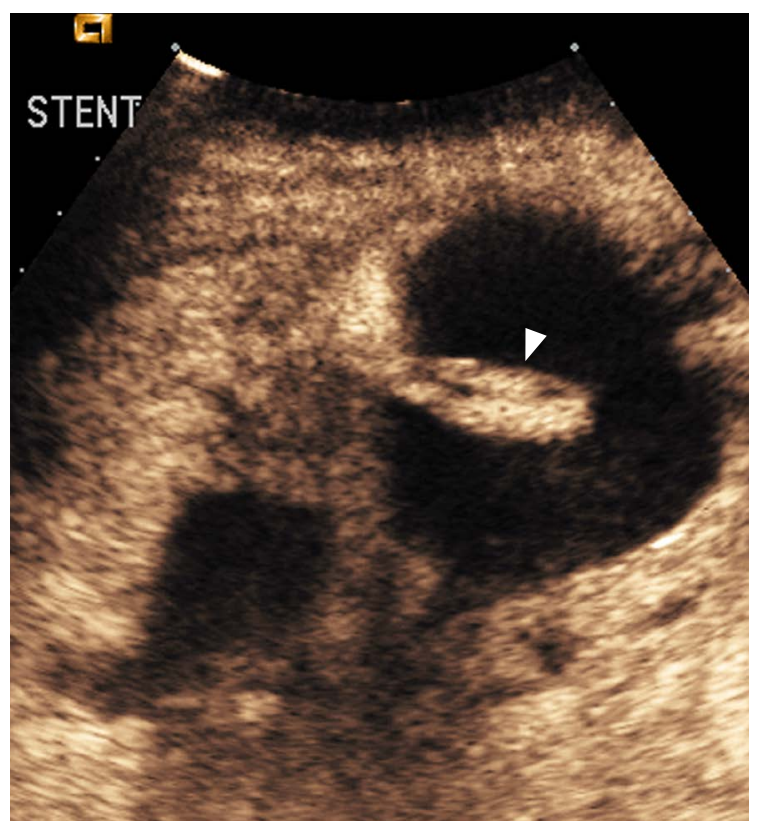

E 


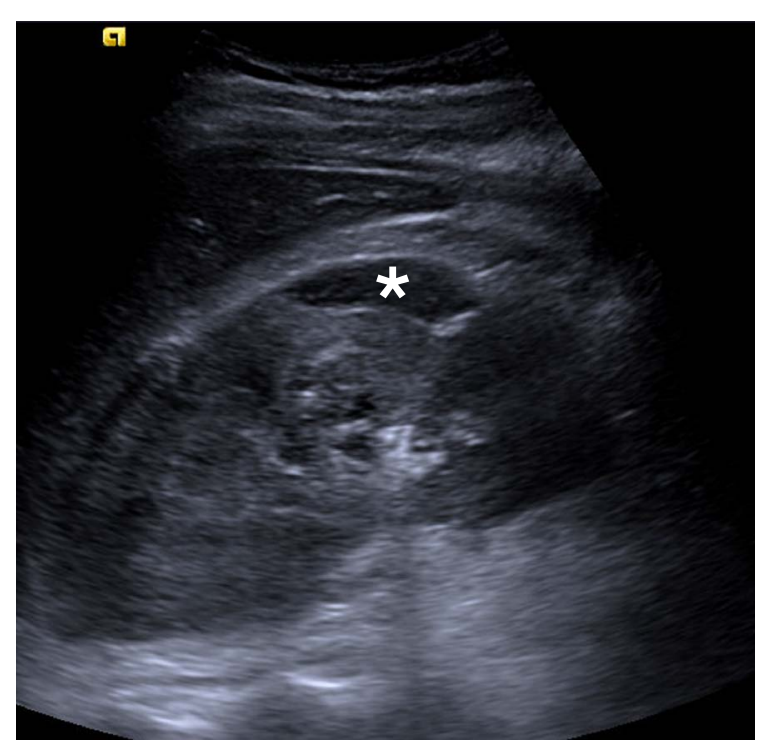

A

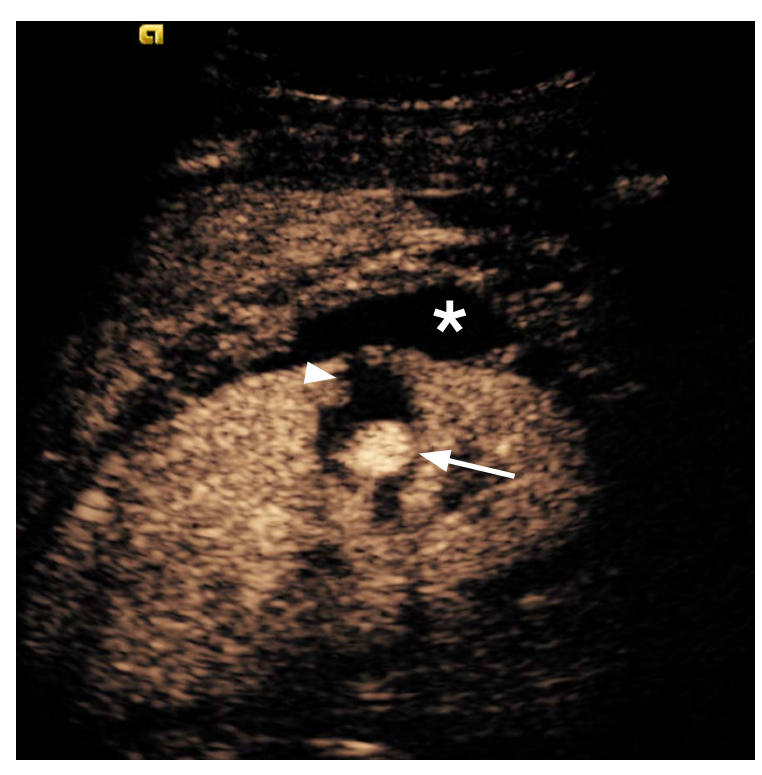

C

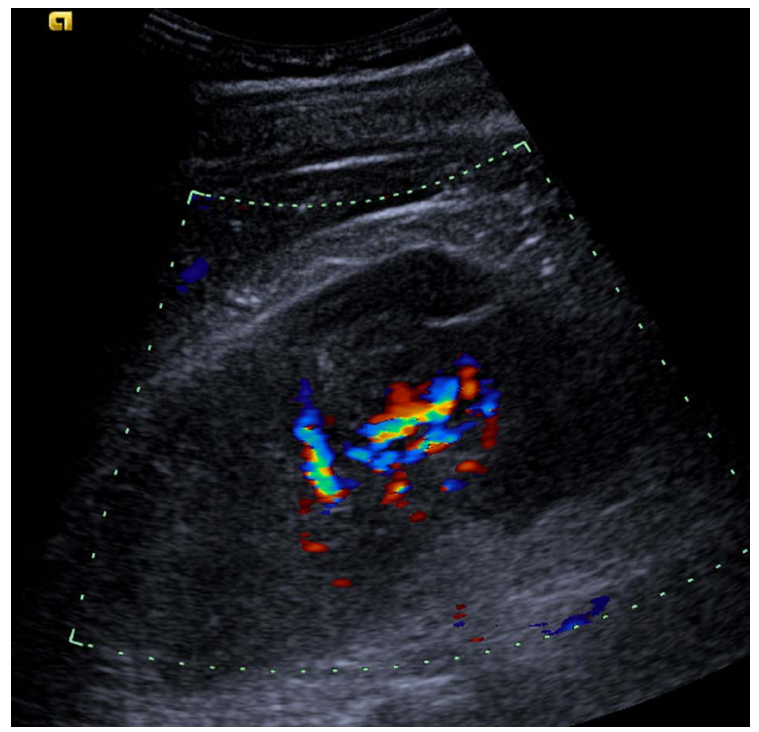

B

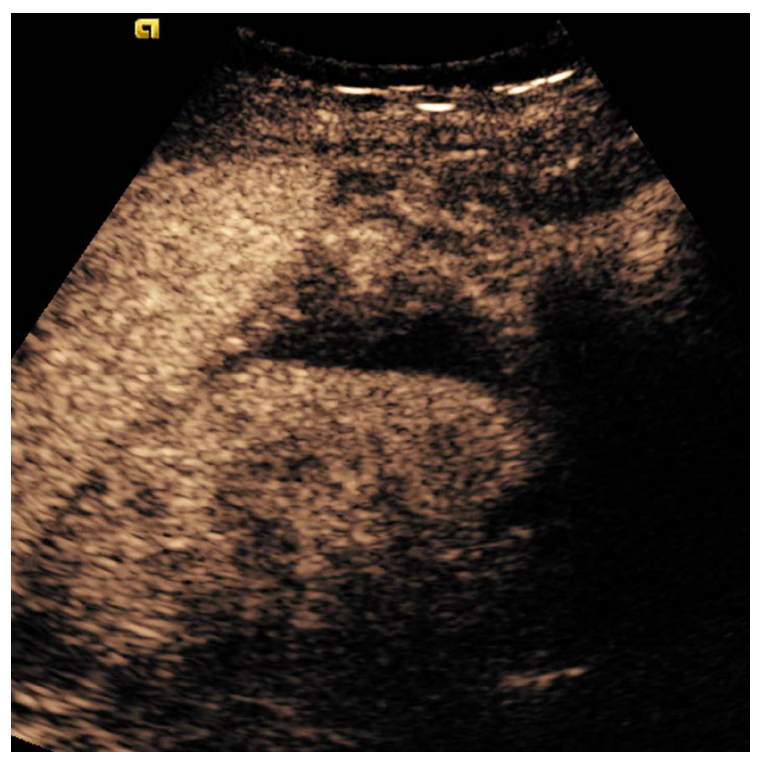

D

Fig. 14. Pseudoaneurysm formation after renal biopsy.

A. A B-mode image shows a subcapsular renal haematoma (asterisk). B. Color Doppler US demonstrates blood flow signals in the renal hilum, with no clear evidence of further pathology. C. A CEUS image demonstrates a pseudoaneurysm (arrow), whilst the subcapsular haematoma (asterisk) and an intra-parenchymal haematoma (arrowhead) become prominent due to a lack of enhancement. D. Followup CEUS image after embolization of the pseudoaneurysm shows absence of the pseudoaneurysm and normal perfusion of the renal parenchyma, confirming the success of the intervention.

thrombus characterization, providing promising results and thus being included in official recommendations. Nonetheless, it has many more potential applications that have not yet been thoroughly studied.

ORCID: Vasileios Rafailidis: https://orcid.org/0000-0002-0284-4106; Dean Y. Huang: https://orcid.org/0000-0002-5637-6723; Gibran Timothy Yusuf: https://orcid.org/0000-
0002-5621-4249; Paul S. Sidhu: https://orcid.org/0000-0003-1928-4077

\section{Author Contributions}

Conceptualization: Rafailidis V, Huang DY, Yusuf GT, Sidhu PS. Data acquisition: Rafailidis V, Huang DY, Yusuf GT, Sidhu PS. Data analysis or interpretation: Rafailidis V, Huang DY, Yusuf GT, Sidhu PS. Drafting of the manuscript: Rafailidis V. Critical revision of the manuscript: 
Huang DY, Yusuf GT, Sidhu PS. Approval of the final version of the manuscript: all authors.

\section{Conflict of Interest}

PS has received lecture fees from Bracco, Siemens, Samsung, Philips, and Hitachi. The rest of the authors have no conflict of interest regarding the publication of this manuscript.

\section{Acknowledgments}

VR has received a scholarship for his PhD studies on "Imaging of the carotid vulnerable plaque with contrast-enhanced ultrasound and multi-detector computed tomography angiography" from the Alexander S. Onassis Public Benefit Foundation. VR has received honorarium from the Korean Society of Ultrasound in Medicine.

\section{References}

1. Sidhu PS. Multiparametric ultrasound (MPUS) imaging: terminology describing the many aspects of ultrasonography. Ultraschall Med 2015;36:315-317.

2. Rafailidis V, Sidhu PS. Vascular ultrasound, the potential of integration of multiparametric ultrasound into routine clinical practice. Ultrasound 2018;26:136-144.

3. Gramiak R, Shah PM. Echocardiography of the aortic root. Invest Radiol 1968;3:356-366.

4. Greis C. Technology overview: SonoVue (Bracco, Milan). Eur Radiol 2004;14 Suppl 8:P11-P15.

5. Piscaglia F, Nolsoe C, Dietrich CF, Cosgrove DO, Gilja OH, Bachmann Nielsen $\mathrm{M}$, et al. The EFSUMB guidelines and recommendations on the clinical practice of contrast enhanced ultrasound (CEUS): update 2011 on non-hepatic applications. Ultraschall Med 2012;33:33-59.

6. Sidhu PS, Cantisani V, Dietrich CF, Gilja OH, Saftoiu A, Bartels $E$, et al. The EFSUMB guidelines and recommendations for the clinical practice of contrast-enhanced ultrasound (CEUS) in nonhepatic applications: update 2017 (short version). Ultraschall Med 2018;39:154-180.

7. Dietrich CF, Averkiou M, Nielsen MB, Barr RG, Burns PN, Calliada $\mathrm{F}$, et al. How to perform Contrast-Enhanced Ultrasound (CEUS). Ultrasound Int Open 2018;4:E2-E15.

8. Chung YE, Kim KW. Contrast-enhanced ultrasonography: advance and current status in abdominal imaging. Ultrasonography 2015;34:3-18.

9. Greis C. Technical aspects of contrast-enhanced ultrasound (CEUS) examinations: tips and tricks. Clin Hemorheol Microcirc 2014;58:89-95.

10. Piscaglia F, Bolondi L; Italian Society for Ultrasound in Medicine and Biology (SIUMB) Study Group on Ultrasound Contrast Agents. The safety of Sonovue in abdominal applications: retrospective analysis of 23188 investigations. Ultrasound Med Biol 2006;32:1369-1375.

11. Tang C, Fang K, Guo Y, Li R, Fan X, Chen P, et al. Safety of sulfur hexafluoride microbubbles in sonography of abdominal and superficial organs: retrospective analysis of 30,222 cases. J Ultrasound Med 2017;36:531-538.

12. Kitzman DW, Goldman ME, Gillam LD, Cohen JL, Aurigemma GP, Gottdiener JS. Efficacy and safety of the novel ultrasound contrast agent perflutren (definity) in patients with suboptimal baseline left ventricular echocardiographic images. Am J Cardiol 2000;86:669674.

13. Cochran ST, Bomyea K, Sayre JW. Trends in adverse events after IV administration of contrast media. AJR Am J Roentgenol 2001;176:1385-1388.

14. Hunt CH, Hartman RP, Hesley GK. Frequency and severity of adverse effects of iodinated and gadolinium contrast materials: retrospective review of 456,930 doses. AJR Am J Roentgenol 2009;193:1124-1127.

15. Main ML, Ryan AC, Davis TE, Albano MP, Kusnetzky LL, Hibberd M. Acute mortality in hospitalized patients undergoing echocardiography with and without an ultrasound contrast agent (multicenter registry results in 4,300,966 consecutive patients). Am J Cardiol 2008;102:1742-1746.

16. Kusnetzky LL, Khalid A, Khumri TM, Moe TG, Jones PG, Main ML. Acute mortality in hospitalized patients undergoing echocardiography with and without an ultrasound contrast agent: results in 18,671 consecutive studies. J Am Coll Cardiol 2008;51:1704-1706.

17. Yusuf GT, Sellars ME, Deganello A, Cosgrove DO, Sidhu PS. Retrospective analysis of the safety and cost implications of pediatric contrast-enhanced ultrasound at a single center. AJR Am J Roentgenol 2017;208:446-452.

18. Marshall MM, Beese RC, Muiesan P, Sarma DI, O'Grady J, Sidhu PS. Assessment of portal venous system patency in the liver transplant candidate: a prospective study comparing ultrasound, microbubbleenhanced colour Doppler ultrasound, with arteriography and surgery. Clin Radiol 2002;57:377-383.

19. Eckersley RJ, Chin CT, Burns PN. Optimising phase and amplitude modulation schemes for imaging microbubble contrast agents at low acoustic power. Ultrasound Med Biol 2005;31:213-219.

20. Forsberg F, Liu JB, Burns PN, Merton DA, Goldberg BB. Artifacts in ultrasonic contrast agent studies. J Ultrasound Med 1994;13:357365.

21. Dietrich CF, Ignee A, Hocke M, Schreiber-Dietrich D, Greis C. Pitfalls and artefacts using contrast enhanced ultrasound. Z Gastroenterol 2011;49:350-356.

22. Forsberg F, Piccoli CW, Merton DA, Palazzo JJ, Hall AL. Breast lesions: imaging with contrast-enhanced subharmonic US: initial experience. Radiology 2007;244:718-726.

23. Forsberg F, Goldberg BB, Liu JB, Merton DA, Rawool NM. On the 
feasibility of real-time, in vivo harmonic imaging with proteinaceous microspheres. J Ultrasound Med 1996;15:853-860.

24. Eisenbrey JR, Daecher A, Kramer MR, Forsberg F. Effects of needle and catheter size on commercially available ultrasound contrast agents. J Ultrasound Med 2015;34:1961-1968.

25. Wilson SR, Jang HJ, Kim TK, lijima H, Kamiyama N, Burns PN. Realtime temporal maximum-intensity-projection imaging of hepatic lesions with contrast-enhanced sonography. AJR Am J Roentgenol 2008; 190:691-695.

26. Nakamura J, Nakamura T, Deyama J, Fujioka D, Kawabata K, Obata $J E_{\text {, et }}$ al. Assessment of carotid plaque neovascularization using quantitative analysis of contrast-enhanced ultrasound imaging is useful for risk stratification in patients with coronary artery disease. Int J Cardiol 2015;195:113-119.

27. Jung EM, Rennert J, Fellner $C$, Uller $W$, Jung $W$, Schreyer $A$, et al. Detection and characterization of endoleaks following endovascular treatment of abdominal aortic aneurysms using contrast harmonic imaging (CHI) with quantitative perfusion analysis (TIC) compared to CT angiography (CTA). Ultraschall Med 2010;31:564-570.

28. Clevert DA, Gurtler VM, Meimarakis G, D'Anastasi M, Weidenhagen $\mathrm{R}$, Reiser MF, et al. Classification of endoleaks in the follow-up after EVAR using the time-to-peak of the contrast agent in CEUS examinations. Clin Hemorheol Microcirc 2013;55:183-191.

29. Fetzer DT, Rafailidis V, Peterson C, Grant EG, Sidhu P, Barr RG. Artifacts in contrast-enhanced ultrasound: a pictorial essay. Abdom Radiol (NY) 2018:43:977-997.

30. ten Kate GL, Renaud GG, Akkus Z, van den Oord SC, ten Cate FJ, Shamdasani $V$, et al. Far-wall pseudoenhancement during contrastenhanced ultrasound of the carotid arteries: clinical description and in vitro reproduction. Ultrasound Med Biol 2012;38:593-600.

31. van den Oord SC, Renaud G, Bosch JG, de Jong N, van der Steen AF, Schinkel AF. Far wall pseudo-enhancement: a neglected artifact in carotid contrast-enhanced ultrasound? Atherosclerosis 2013:229:451-452.

32. Gutberlet M, Venz S, Neuhaus R, Ehrenstein T, Lemke AJ, Vogl TJ, et al. Contrast agent enhanced duplex ultrasonography: visualization of the hepatic artery after orthotopic liver transplantation. Rofo 1997;166:411-416.

33. Gutberlet M, Venz S, Zendel W, Hosten N, Felix R. Do ultrasonic contrast agents artificially increase maximum Doppler shift? In vivo study of human common carotid arteries. J Ultrasound Med 1998; 17:97-102.

34. Sirlin CB, Lee YZ, Girard MS, Peterson TM, Steinbach GC, Baker $K G$, et al. Contrast-enhanced B-mode US angiography in the assessment of experimental in vivo and in vitro atherosclerotic disease. Acad Radiol 2001;8:162-172.

35. Kono Y, Pinnell SP, Sirlin CB, Sparks SR, Georgy B, Wong W, et al. Carotid arteries: contrast-enhanced US angiography: preliminary clinical experience. Radiology 2004;230:561-568.
36. Lee KW, Park YJ, Rho YN, Kim DI, Kim YW. Measurement of carotid artery stenosis: correlation analysis between B-mode ultrasonography and contrast arteriography. J Korean Surg Soc 2011;80:348-354.

37. Ten Kate $G L$, van den Oord SC, Sijbrands EJ, van der Lugt $A$, de Jong N, Bosch JG, et al. Current status and future developments of contrast-enhanced ultrasound of carotid atherosclerosis. J Vasc Surg 2013;57:539-546.

38. Ventura CA, Silva ES, Cerri GG, Leao PP, Tachibana A, Chammas MC. Can contrast-enhanced ultrasound with second-generation contrast agents replace computed tomography angiography for distinguishing between occlusion and pseudo-occlusion of the internal carotid artery? Clinics (Sao Paulo) 2015;70:1-6.

39. Hammond CJ, McPherson SJ, Patel JV, Gough MJ. Assessment of apparent internal carotid occlusion on ultrasound: prospective comparison of contrast-enhanced ultrasound, magnetic resonance angiography and digital subtraction angiography. Eur J Vasc Endovasc Surg 2008;35:405-412.

40. Clevert DA, Sommer WH, Zengel P, Helck A, Reiser M. Imaging of carotid arterial diseases with contrast-enhanced ultrasound (CEUS). Eur J Radiol 2011;80:68-76.

41. ten Kate GL, Sijbrands EJ, Staub D, Coll B, ten Cate FJ, Feinstein SB, et al. Noninvasive imaging of the vulnerable atherosclerotic plaque. Curr Probl Cardiol 2010;35:556-591.

42. Rafailidis V, Chryssogonidis I, Xerras C, Nikolaou I, Tegos T, Kouskouras $\mathrm{K}$, et al. A comparative study of color Doppler imaging and contrast-enhanced ultrasound for the detection of ulceration in patients with carotid atherosclerotic disease. Eur Radiol 2019;29:2137-2145.

43. ten Kate GL, van Dijk AC, van den Oord SC, Hussain B, Verhagen HJ, Sijbrands EJ, et al. Usefulness of contrast-enhanced ultrasound for detection of carotid plaque ulceration in patients with symptomatic carotid atherosclerosis. Am J Cardiol 2013;112:292-298.

44. Rafailidis V, Chryssogonidis I, Tegos T, Kouskouras K, CharitantiKouridou A. Imaging of the ulcerated carotid atherosclerotic plaque: a review of the literature. Insights Imaging 2017;8:213-225.

45. Staub D, Schinkel AF, Coll B, Coli S, van der Steen AF, Reed JD, et al. Contrast-enhanced ultrasound imaging of the vasa vasorum: from early atherosclerosis to the identification of unstable plaques. JACC Cardiovasc Imaging 2010;3:761-771.

46. Staub D, Patel MB, Tibrewala A, Ludden D, Johnson M, Espinosa $P$, et al. Vasa vasorum and plaque neovascularization on contrastenhanced carotid ultrasound imaging correlates with cardiovascular disease and past cardiovascular events. Stroke 2010;41:41-47.

47. Ritter MA, Theismann K, Schmiedel M, Ringelstein EB, Dittrich R. Vascularization of carotid plaque in recently symptomatic patients is associated with the occurrence of transcranial microembolic signals. Eur J Neurol 2013;20:1218-1221.

48. van den Oord SC, Akkus Z, Renaud G, Bosch JG, van der Steen 
AF, Sijbrands EJ, et al. Assessment of carotid atherosclerosis, intraplaque neovascularization, and plaque ulceration using quantitative contrast-enhanced ultrasound in asymptomatic patients with diabetes mellitus. Eur Heart J Cardiovasc Imaging 2014;15:1213-1218.

49. Kim HS, Woo JS, Kim BY, Jang HH, Hwang SJ, Kwon SJ, et al. Biochemical and clinical correlation of intraplaque neovascularization using contrast-enhanced ultrasound of the carotid artery. Atherosclerosis 2014;233:579-583.

50. van den Oord SC, Akkus Z, Roeters van Lennep JE, Bosch JG, van der Steen AF, Sijbrands EJ, et al. Assessment of subclinical atherosclerosis and intraplaque neovascularization using quantitative contrast-enhanced ultrasound in patients with familial hypercholesterolemia. Atherosclerosis 2013;231:107-113.

51. Rafailidis V, Charitanti A, Tegos T, Destanis E, Chryssogonidis I. Contrast-enhanced ultrasound of the carotid system: a review of the current literature. J Ultrasound 2017;20:97-109.

52. Giannoni MF, Vicenzini E, Citone M, Ricciardi MC, Irace L, Laurito A, et al. Contrast carotid ultrasound for the detection of unstable plaques with neoangiogenesis: a pilot study. Eur J Vasc Endovasc Surg 2009;37:722-727.

53. Xiong L, Deng YB, Zhu Y, Liu YN, Bi XJ. Correlation of carotid plaque neovascularization detected by using contrast-enhanced US with clinical symptoms. Radiology 2009;251:583-589.

54. Faggioli GL, Pini R, Mauro R, Pasquinelli G, Fittipaldi S, Freyrie $A$, et al. Identification of carotid 'vulnerable plaque' by contrastenhanced ultrasonography: correlation with plaque histology, symptoms and cerebral computed tomography. Eur J Vasc Endovasc Surg 2011:41:238-248.

55. Schinkel AF, van den Oord SC, van der Steen AF, van Laar JA, Sijbrands EJ. Utility of contrast-enhanced ultrasound for the assessment of the carotid artery wall in patients with Takayasu or giant cell arteritis. Eur Heart J Cardiovasc Imaging 2014;15:541546.

56. Rafailidis V, Chryssogonidis I, Tegos T, Partovi S, CharitantiKouridou A, Staub D. Role of multi-parametric ultrasound in transient perivascular inflammation of the carotid artery syndrome. Ultrasound 2019;27:77-84.

57. Clevert DA, Sommer WH, Helck A, Reiser M. Duplex and contrast enhanced ultrasound (CEUS) in evaluation of in-stent restenosis after carotid stenting. Clin Hemorheol Microcirc 2011;48:199-208.

58. Li ZJ, Luo XH, Du LF. Identification of carotid artery dissection by contrast enhanced ultrasonograph: a case report. Med Ultrason 2015;17:564-565.

59. Clevert DA, Kubisch C, Meimarakis G, Zengel P, Reiser M. Improved visualization of carotid-jugular arteriovenous fistula by contrastenhanced ultrasound. Ultraschall Med 2010;31:610-612.

60. Catalano O, Lobianco R, Cusati B, Siani A. Contrast-enhanced sonography for diagnosis of ruptured abdominal aortic aneurysm.
AJR Am J Roentgenol 2005;184:423-427.

61. Partovi S, Imfeld S, Aschwanden M, Bilecen D, Jaeger KA, Staub D. The use of contrast-enhanced ultrasound (CEUS) in chronic periaortitis. Ultraschall Med 2013;34:3-6.

62. Negrao de Figueiredo G, Muller-Peltzer K, Schwarze V, Rubenthaler J, Clevert DA. Ultrasound and contrast enhanced ultrasound imaging in the diagnosis of acute aortic pathologies. Vasa 2019;48:17-22.

63. Clevert DA, Stickel M, Johnson T, Glaser C, Clevert DA, Steitz $\mathrm{HO}$, et al. Imaging of aortic abnormalities with contrastenhanced ultrasound. A pictorial comparison with CT. Eur Radiol 2007;17:2991-3000.

64. Clevert DA, Sommer WH, Meimarakis G, Kopp R, Weidenhagen $R$, Reiser M. Contrast-enhanced ultrasound compared with multislice computed tomography for endovascular aneurysm repair surveillance. Ultrasound 2011;19:11-19.

65. d'Audiffret A, Desgranges P, Kobeiter DH, Becquemin JP. Followup evaluation of endoluminally treated abdominal aortic aneurysms with duplex ultrasonography: validation with computed tomography. J Vasc Surg 2001;33:42-50.

66. Sommer WH, Hoffmann RT, Becker CR, Reiser MF, Clevert DA. Comparison of time-resolved CT-angiography, contrast-enhanced ultrasound and digital subtraction angiography in a patient with a small type II endoleak after endovascular aneurysm repair. Clin Hemorheol Microcirc 2010;45:19-25.

67. Li X, Staub D, Rafailidis V, Al-Natour M, Kalva S, Partovi S. Contrastenhanced ultrasound of the abdominal aorta: current status and future perspectives. Vasa 2019;48:115-125.

68. Rafailidis V, Partovi S, Dikkes A, Nakamoto DA, Azar N, Staub D. Evolving clinical applications of contrast-enhanced ultrasound (CEUS) in the abdominal aorta. Cardiovasc Diagn Ther 2018;8 (Suppl 1):S118-S130.

69. Rafailidis V, Fang C, Yusuf GT, Huang DY, Sidhu PS. Contrastenhanced ultrasound (CEUS) of the abdominal vasculature. Abdom Radiol (NY) 2018;43:934-947.

70. Tarantino L, Ambrosino P, Di Minno MN. Contrast-enhanced ultrasound in differentiating malignant from benign portal vein thrombosis in hepatocellular carcinoma. World J Gastroenterol 2015;21:9457-9460.

71. Claudon M, Dietrich CF, Choi BI, Cosgrove DO, Kudo M, Nolsoe CP, et al. Guidelines and good clinical practice recommendations for contrast enhanced ultrasound (CEUS) in the liver: update 2012: a WFUMB-EFSUMB initiative in cooperation with representatives of AFSUMB, AIUM, ASUM, FLAUS and ICUS. Ultraschall Med 2013;34:11-29.

72. Piscaglia F, Gianstefani A, Ravaioli M, Golfieri R, Cappelli A, Giampalma $E$, et al. Criteria for diagnosing benign portal vein thrombosis in the assessment of patients with cirrhosis and hepatocellular carcinoma for liver transplantation. Liver Transpl 2010;16:658-667. 
73. Rossi S, Rosa L, Ravetta V, Cascina A, Quaretti P, Azzaretti A, et al. Contrast-enhanced versus conventional and color Doppler sonography for the detection of thrombosis of the portal and hepatic venous systems. AJR Am J Roentgenol 2006;186:763-773.

74. Tarantino L, Francica G, Sordelli I, Esposito F, Giorgio A, Sorrentino $P$, et al. Diagnosis of benign and malignant portal vein thrombosis in cirrhotic patients with hepatocellular carcinoma: color Doppler US, contrast-enhanced US, and fine-needle biopsy. Abdom Imaging 2006;31:537-544.

75. Chammas MC, Oliveira AC, D'Avilla MJ, Moraes PH, Takahashi MS.
Characterization of malignant portal vein thrombosis with contrastenhanced ultrasonography. Ultrasound Med Biol 2019;45:50-55.

76. Huang DY, Yusuf GT, Daneshi M, Ramnarine R, Deganello A, Sellars $M E$, et al. Contrast-enhanced ultrasound (CEUS) in abdominal intervention. Abdom Radiol (NY) 2018;43:960-976.

77. Durkin N, Deganello A, Sellars ME, Sidhu PS, Davenport M, Makin E. Post-traumatic liver and splenic pseudoaneurysms in children: diagnosis, management, and follow-up screening using contrast enhanced ultrasound (CEUS). J Pediatr Surg 2016;51:289-292. 